\title{
JOHN CAPGRAVE \\ UND DIE ENGLISCHE SCHRIFTSPRACHE. $\left.{ }^{1}\right)$
}

\section{Vierter teil: Formenlehre.}

\section{Substantivum.}

1. Genetiv.

(§ 217) Bei Capgrave und in den Paston Letters ist der genetiv in vielen fällen endungslos. Zunächst fehlt, wie oft im Me., die kasusendung bei den verwandtschaftsnamen, so his fader blessyng Chr. 29 (doch his fadirs scheep 39), in his modir wombe 46 usw., moder gen. : other Kath. I 753, undre my moodre seall 2JP III 103, hyr broder advice MP II 26, myn unkyll masagear (Bote) * III 221 usw. Ferner werden ebenso behandelt (ganz oder z. t. unter afz. einfluss, vgl. Einenkel in Pauls Grundriss $\mathrm{I}^{2} \S 142 \gamma$ ) 1. Eigennamen, vgl. Tubal hamberes Chr.8, Philippe, Alisaundre brothir 53, Jozabeth, the Kyngis dowtir Joram 41, the name of Abraham frend 11, the chaumbir of Constantin dowtir 80, Herbert (Gen.) partye Wo I (417), Heydon son MP II (125) - doch Dame Elyzabeth Brewsys lettyr 3JP III 198. 2. Titel: the emperoure moder $\mathrm{Chr} .70$, the duke doutir of Normandie 127, the duke of Ostrich men 146, the Pope bridil 103, the King hed 133, the erle doutir 175, the Abbot chambir 187, on the qween (doch ae. gen. cwene) side 195, - the Duc men Norf. II (205), the Kynge lawys 2WiP III 259, the Kyng wyffe Wo I (417), my maister evidences, yn my maister name Wo I 370 usw. 3. Andere appellativa: a carter child Chr.

1) Fortsetzung aus band XXIII heft II s. 153-194, heft III s. 323-375 und heft IV s. $427-472$ dieser zeitschrift. 
186, a dogge tail 281, the castelle wallis 122 , upon a day warnyng PL * III 354. Die letzten fälle sind fast schon als zusammensetzungen zu betrachten. Ferner at ye aldermannes wyl and at ye cumpany (gen.) Norf. gilden 30 , he ... come unto a servaunt hous of myn ... cald William Shirref Linc. PL I (97).

Ausserhalb Norfolk sind derartige fälle endungslosen genetivs in unseren quellen selten (our lady lyght $\mathrm{u}$. ä. bei Morsbach s. 111 beruht auf alter genetivendung $-e$; seint Katerine day u. ä. (ebd.) wird unter lateinischem einfluss daran angeglichen sein). Mir sind aufgefallen the fadre lawis 2 Makk. VII $2 \mathrm{a}$ und in den urkunden Richards III afore the king coronacion 24, the king .. grace 71 . Beispiele aus dem 16. jahrhundert s. Hoelper 47.

\section{Plural.}

(§ 218) In einigen auf einen zischlaut ausgehenden wörtern (meist romanischen ursprungs) ist die pluralendung $-s$ mit dem auslautenden konsonanten des stammes verschmolzen. So erscheint in London wytnesse (Morsbach 113), paresshe W 114/25, in Suffolk vers 1467: 19, in Norfolk ordinance (Schultz 31), in Kath. in dyuerse place : in hys ... place II 649, the seuyn scyens : expens (sg.) : dylygens (sg.) III 212, sacryfise (pl.) : despise IV 742 - ebs. places : was Gen. 1467, Lon. XXII 78, in Chr. liknes 33, chalis 146, place 164, 190, 268, sciens 104, 110,113, (doch sciensis 10, expenses 230 ), vers 133, 173, 221 u. ö., in den PL on of the justice of pease Wo I 370, bei Caxton plays (frz. sing. plais $=$ ne. plaice), fyssh $(e) 26,95$ neben fysshes 96. Vgl. ne. plur. fish, verse, die jedoch auch andere erklärungen zulassen.

Doppelter plural liegt vor in grecis $<$ gré $<$ gradum Chr. 78, gresys Suff. 1467: 20.

(§ 219) Eine beträchtliche anzahl von schwachen substantiven hat die ae. pluralendung $-n$ bewahrt. Einige belege aus den werken der Chaucerschüler mögen genügen: foon : anoon Hoccleve MP 91/466, Lydgate AG 1762, floon (ae. flān pfeile) : goon MP 259/468, been (zu ae. béo biene) : queen Capgrave, Kath. IV 255, schon Chr. 70, oxen 83, hosen 70, eyne 31, 78. Auch einige wörter, die ursprünglich nicht schwach flektiert wurden, haben den $n$-plural angenommen, vgl. bretherin $\mathrm{Chr}$. 
108, 158 u. ö., childirn Chr. 16, 18, 27 neben chitair 17, 43. So auch children Hos. IX $12 \mathrm{~b}$, XIV $1 \mathrm{~b}$, Micha II $9 \mathrm{~b}$, Mark. X 14 (childre Hos. IX 12 a, XIV 1 a, Micha II 9a), lambren Dan. III 40, Luk. X 3, fadryn Gal. I 14 (b: fadris), kijn $<k \bar{y}$ kühe Hosea X 5, kyen Amos IV 1, kijn Pecock 259, kyen R 69, brothern, brederyn Exeter 335, 336, bretheren, childrin, eyren (Morsbach 114), bretheryn 2JP III 139 u. ö., brodyrn WaP III 226 (brether MP III 135), so auch knyghtenspence (gen. plur.!) Worcester 390. Doppelte pluralbildung liegt vor in wyndownes (an. windauga > window!) 1JP I 252, * III 211.

In ne. mundarten bilden noch andere starke substantiva den plural auf -n, vgl. hauzn u. ä. Ellis 48, 119, 271 u. ö., tlözzn (ae. clāp) 220, cheesen (ae. ciese) 271, sogar plēzn (frz. place) 60, 212.

(§ 220) Bei einigen starken neutris ist in ae. weise der plural dem singular gleich. Für die Chaucerschule mögen genügen die angaben von $\mathrm{t}$. $\mathrm{Br}$. ( $\$ 206)$, ferner folgende beispiele: schepe : kepe Hoccleve RPr 1428, yeer : cheer Lydgate, Giles 106, ebs. EFr II 987, III 1435, AA I 707, swyn : myn Capgrave IV 1480, ping : kynge Pr 33 (aber thyngis : kyngis I 688 u. ö.), zeere : here IV $1877 \mathrm{u}$. ö., und aus Chr. hors 255, 256, $292 \mathrm{u}$. ö., pound 89, 130, 195 u. ö., thing 194, 293, 305 u. o. (thingis 1, 20, 305 u. ö.), zere 13, 27, 29 u. ö. (zeres 13, 63 u. ö)., lite < lēoht 22 (?), tre 21 (trees 9, 34, 35 u. ö.), scheep) 8, 31, 39 u. ö., wynter 29, 35, dazu wynter Morsbach 114.

(§ 221) Bei massbestimmungen hat sich aus dem von zahlwörtern abhängigen genetiv ein endungsloser plural entwickelt; auch analogie $\mathrm{zu}$ wörtern wie pund, zeār konnte wirksam werden, sobald der genetiv nicht mehr als solcher gefïhlt wurde. So hat Bokenam myle plur. : begyle Pr. 113, ebs. XII 82, Capgrave fote 197 , myle 103, 268, score 13, 15, 96, sithe 3 (sithis ebd.), tunne 239, in den Londoner urkunden erscheint mile (Morsbach 113), in Norfolk mile, pound(e), zere, skore, ston (Schultz 31), in den Paston Letters boshell 3JP III $7 \mathrm{u}$. ä.

Auch andere wörter finden sich oft ohne pluralbezeichnung. Teilweise können die endungslosen formen reste ae. deklinationsarten sein, so broder (Ms. e) : oder (Ms.: othyr) Audelay 35, doughtyr 3JP III 82, brethere, systere, brothere, modere, dazu fadere (Schultz $31 \mathrm{f}$ ), goot (ae. gōt mit beeinflussung durch den singular?) Chr. 8 , hethen $<$ hōpne (adj.) 
Chr. 82, monthe < ae. plur. mōnap Morsbach 114, morunspeche (ae. plur. spräce) Schultz 31, auch candelle (ebd.)?, ferner chyldyr u. ä.; vgl. § 219 und 3JP III 150, 197, 289 u. ö.; meist jedoch wird analogie der substantive gewirkt haben, bei denen beide numeri gleich lauteten, so in auter Chr. 15, puple 17, 21, 40 u. oft (vgl. ne. people; puples = völker 18), electoure 158, alye 286, crym 265. Aus Caxtons RF ist zu erwähnen membre 44 (druckfehler?).

In dede of armes Chr. 33 liegt gruppenplural vor. - Bemerkenswert ist noch $a$ (sing.) sufficient amendis (plur. $<$ frz. amendes Pecock 110.

\section{Geschlecht}

(§ 222) Ae. feminina und neutra erscheinen nicht selten als maskulina, so bei Capgrave lond (neutr.) als maskulinum 23, bei Pecock die feminina scoling schulung 90, book 4 u. ö., lawe 70, treuthe 99 u. ö., deedis 123, roode 194 (neutr. 223), brigge 338, world 548 und die neutra thing 17, hous 11, writt 16, Ynglond 86, dazu mankinde 74 (ae. cynde wird als $\mathrm{n}$. und f. gebraucht). - Männliches geschlecht zeigen ferner die fremdwörter argument Pecock $102 \mathrm{u}$. oft, clergie 2, article 53, text 64, crosse 199 , reward 384 , couent 346 , auch scripture $35 \mathrm{u} .00$. (neutrum 73), conclusioun 43, opinioun 96, noumbir $\mathrm{Chr} .2$; als weiblich betrachtet Capgrave cyte 160 , als männlich bereits sunne 44.

\section{Adjektivum.}

(§ 223) In den meisten unserer prosadenkmäler erscheint gelegentlich die frz. pluralendung $-s$, jedoch fast nur bei romanischen wörtern. Chaucer hat noumbres proporcionables $u$. ä. (t. Br. $\$ 243$ ), Capgrave many justeres (zu frz. jouste) alienes Chr. 175, die Londoner urkunden alle pe goodis pat be meuablis $\mathrm{W} 80 / 5$, I paire candelstekes secundaries $82 / 2$, many wronges subtiles M 2, die Norfolker gilden sufficiauntz borwes BL 92, die verfassung von Worcester other (diuers) yeld(e)s precedents 379, 402. Die Paston Letters bieten den frz. plural auch bei vorgestelltem adjektiv, so certeins notables and resonables causes $1 \mathrm{WiP} I$ 30, at diverses tymes * II (66), the Kings moste noblez lettrez, in many diversez bihalvez ${ }^{*} \mathrm{I}(421)$, sogar bei einem germanischen eigenschaftswort: my goods Maysters * II (298); 
ähnlich yours goode cosynes and frendes ${ }^{*} \mathrm{I} 209$. Caxton (Römstedt 38) hat frz. plural nur in romanischen wörtern.

Die ae. endung des gen. plur. ist noch erhalten in God our aller Creatour R 53.

(§ 224) Der im Ae. nicht seltene $i$-umlaut im komparativ und superlativ der adjektiva hat sich im 15. jahrhundert nur noch bei eald, long, und strong erhalten, jedoch sind neben den umgelauteten auch nicht umgelautete formen im gebrauch. In der Bibel finden wir stets elder, stronger und strenger (belege bei Gasner s. 77 f.), meist lengere, lenger, lengre 2 Makk. XII 36, Mark. XII 40a, Luk. XXII 43, 1 Thess. III 1, ausnahmsweise auch longer Mark. XII 40 b. Pecock schreibt einmal longer 223, sonst bedient er sich stets der umgelauteten formen, vgl. lengir u. ä. 15, 64, 78 u. oft, lengist 133, strenger 46, 77, strengist 527, eeldir 106, 107, 108 u. ö. (aldir men 215), eeldist 118, 133, 249 u. ö. Auch Chaucer hat umlaut in diesen formen (t. Br. § 244), ebenso gewöhnlich Capgrave: elder 8, 131, 187 u. ö., eldest 78, 107, 206 u. ö., lenger 44, 121, 265 u. ö., longer 51, 110 , strenger 73, 289. Aus den Londoner urkunden führt Morsbach (s. $117 \mathrm{f}$.) an elder, eldest, strenger, lenger, longer, - lenger erscheint auch in den späteren staatsurkunden ( $R$ 44). In den Suffolker testamenten sind $\mathrm{zu}$ belegen theldere 1481: 65, ebs. 1482: 54, theldest 1481: 66, oldest 1467: 24, longere 1467: 36 ; in den Norfolker gilden lenger, lengest, einmal longer (Schultz 32), dazu lenger 1459 I 461 . In den Paston Letters sind beide arten der steigerung im gebrauch, vgl. elder MP II (125), 239, Wo II (313), older MP II 187, 212 usw. - noch kein unterschied zwischen elder und older - lenger $\mathrm{u}$. ä. $1 \mathrm{WiP}$ I 30, 2WiP II 109, MP III 134 u. ö., 2JP III 116 u. ö., 3JP II (305), longer u. ä. 2WiP II 108 u. ö., MP II (362 u. ö.), 2JP III (89), lengest MP I (250), longest 3.JP III 180, strenger MP II 216, strengest Norf. II (68). Der positiv streng Wo I (417) braucht nicht neubildung nach strenger, strengest $\mathrm{zu}$ sein, sondern kann auf ae. strenge beruhen. Dagegen ist das adverbium des positivs leng(e) MP II $(290,291)$ neubildung nach dem komparativadverbium lenge (ebd. 291). Bei Caxton ist umlaut noch recht gebräuchlich (Römstedt 39).

(§ 225) Die kürzung der langen vokale im komparativ und superlativ ist in unseren quellen noch üblich. Wycliffe bietet gretter, grettest (Gasner 77), lennere < hlōene Dan. I $10 \mathrm{~b}$, hatter 
Dan. III 19, brodder Ezech. XIVII 6, XLI 7 (b: braddere), sunner 1 Makk. II 40 usw., Pecock bbroddir 80, deppir 159, gretter 158, 159, 213 u. ö., swetter, swetttist 67, widdir 347 (wijdir ebd.), Chaucer gretter, grettest, derrre (t. Br. § 244), Capgrave gretter 214, grettest 100, 265, die Loondoner urkunden gretter, grettest, derrest (Morsbach 117 f.), spätter derrest $\mathrm{E} \mathrm{12,} \mathrm{29,} 57$ u. ö. neben derest 46, sonnest $\mathrm{R} 72$, idie Suffolker testamente grettere 1467: 20, 23, 29, die Paston Letters gretter 2JP III 199, 3JP III 267, Wo I 404 u. ö., grettist u. ä. Wo I 370, 1JP I 276, MP II 195 usw., nerrest MP II 241, ¿2JP III 199, seltener greter, gretest Wo I 370, MP III 126 u. ö.., Caxton grettest, deppest (Römstedt 39$)$.

(§ 226) Steigerung eines, adjektivischen partizipiums liegt vor in the louyngist Pecock. 130. Doppelte steigerung zeigt sich in furtherer F I (376), mnore hastyer remedy Worcester 377, the most abilist persons Exetter 335, as moste beste can be advysed Suffolk 1482: 54, vgll. dazu most best and expedient in Norfolk, das Schultz 232 ohhne grund verbessern will. Aehnliche fälle bei Caxton (Römsstedt 39).

\section{AAdverbium.}

(§ 227) Ueber ae. -līce vggl. § 198.

Hier ist zu erwähnen ddas nördliche till, das auch in unseren quellen bisweilen für das südlich-mittelländische to eintritt. til to findet sich einigge male in der handschrift $\mathrm{K}^{2} \mathrm{der}$ Bibel (Apg. XVII 14, XXI 55, XXIII 23 u. ö.), til in den werken der Chaucerschule (tylle : ffulfille Nun 29, : wylle Wade 1629, Bokenam I 1122, : spylle Cंuapgrave II 481), häufiger in der volkstümlichen litteratur (: w wille Lon. XXIII 554 u. ö., Cobsam 583, : fulfill Beryn 3972 u. ö.., : stille Unz. T. 219, : wylle vMarg. 272, Wolf. 152, Stat. Jer. 2663, Lamb. II 11/103 u. ö., Brompton 22, Ryman LXXXIV 5 u. ö.j., : fulfyl Audelay 80, : hylle usw. PlSa 195). Aus der prosa ssind zu erwähnen till PL * II (420), tyl Caxton BE 34,1,49, ${ }_{19}, 566,7,57,,_{22}, 69,_{16}$ usw.

(§ 228) Neben dem kompparativadverbium further, forther $<$ furbor findet sich häufiger' eine durch ae. feorr beeinflusste form ferther, vgl. ferther u.l. ä. 2 Tim. III 9, Pecock 2, 3, 53 u. oft (forther nur 12, 345j, 403), Capgrave Chr. 188, 195, Morsbach 35, R 16, 23, 58 uu. ö. (forthre 17, furthre 46), Suffolk 1467: 26, 2WiP I 86, 2 JP III 199, 3JP III 40, MP II $25-$ 
forther EIP II $(330,331)$, Norf. III (255) - , ferthest MP II 82, 2JP III 86, 3JP III 21 usw., forthihest 3JP III 12 u. ö., ffurdest * II (248); dazu ein positiv ferthe : MP II 20, 282. Caxton hat ferther 80, 87, 95 u. oft, further 1109, forther 27.

\section{Zahlwwort.}

(§ 229) Der geschlechtsuntersckhied von ae. twēgen und $t w \bar{a}$ ist im 15. jahrh. bereits beseitigt. . In der Bibel ist two in $\mathrm{K}^{1}$ und $\mathrm{M}$ (auch $\mathrm{A}$ ) herrschend gewoorden, twey(e), tweyne in $\mathrm{K}^{2}$ und b (Gasner $118 \mathrm{ff}$.). Pecock sschreibt twey proposiciouns 8, tweyne pointis 15, these tweinae (diese zwei dinge) 286; Chaucer gebraucht twey $(n) e$ und twwo durcheinander (t.Br. §247), Capgrave nur to(o), vgl. too menn 14, 39, kyngis 38, prestis 72 usw.; brigges 105, dowteres 2006; zere 12, childirn 43; die Londoner und Norfolker urkundeen verwenden twey und two ohne unterschied (Morsbach 118, : Schultz 32); in den übrigen quellen wird two fast ausschliessislich gebraucht (aber tweyn hogges Suff. 1470: 46); doch findett sich tweyne noch bei Caxton (Römstedt 39).

(§ 230) Substantivisches ae. opeer hat noch kein plural-s angenommen, vgl. Luk. XXIV 9, Peecock 5, 134, 135 u. ö., t. Br. $\S 249$ und für Capgrave other : foother Kath. I 57, othir 1, 46, 73 u. ö., ferner R 13, 24, Suffolk 14637: 25, Norfolk 1459 I 460 u. ö., ebs. Hoelper 48, nur ausnahmsweisise othyrs Wo I 371.

(§231) Bei den zahlen von virier aufwärts lautete im Ae. die endung der ordinalia je nach dder natur des vorhergehenden lautes bald $-b a$, bald $-t a$. Im Mde. wurde dieser unterschied jedoch nicht immer beobachtet; teeils -the, teils -te werden oft verallgemeinert.

Wycliffe hängt stets die enduung -the an den positiv, vgl. forthe Luk. III 19 a, fourthe Sach. IVII 1, Luk. IX 7 a, Off. IV 7 , VIII 12 u. ö., fifthe (b: fyuethe) Ezecch. VIII 1, XX 1, XXXIII 21, Sach. VII 3, fyuethe Off. VI 9, IXX 1, XXI 19, seuenthe Joh. IV 52, Judas 14, Off. VIII 1, Ezech. XX 1 u. ö., ciztthe 2 Petr. II 5 (b: eizthe), Off. XVII . 11 u. ö., eiztethe Luk. I 59 a, nynthe Sach. VII 1, Luk. XXIII 44, Apg. III 1, X 3 u. ö., tenthe Joh. I 39, Off. XI 13, Ezecch. XX 1 u. ö., eleuenthe Off. XXI 20, elleuenthe (b: enleuenthe)) Ezech. XXVI 1, XXX 20, 
XXXI 1 u. ö., twelueth(e) Ezech. XXIX 1, XXXII 1, tweluthe Off. XXI 20 (b: tweluethe), twelfthe XXXIII 21 (b: tweluethe) usw., thrittenthe (b: thrittethe) 2 Makk. XV 37, fourteneth (b: -tenthe) Apg. XXVII 27, fiftenthe Ezech. XLV 25 usw., ausgenommen eizt(e) Sach. I 1 a, Luk. I $59 \mathrm{~b}$, Apg. VII $8 \mathrm{~b}$ und stets sixte Luk. XXIII 44, Apg. X 9, Off. VI 12, Ezech. VIII 1 u. ö. Bei Pecock sind nur fifthe 524 und seuenthe $475 \mathrm{zu}$ belegen; Chaucer (t. Br. $\S 429$ ) scheint den ae. unterschied zu bewahren, er hat einerseits ferthe, tenthe, andrerseits fifte, sixte. Bei Capgrave dagegen ist das suffix -t $a$ allein herrschend geworden, vgl. fourt(e) 4, 10, 36 u. ö., fift(e) 36, 95, 136 u. ö., sexte 10 , 95,96 u. ö., sevenet 238 , tent 266 . In Kath. finde ich nur den reim feerde : erthe V 7. Aus den Londoner urkunden führt Morsbach (s. 119) an fourpe, sixte, sixthe, eyghte, tenth; dazu

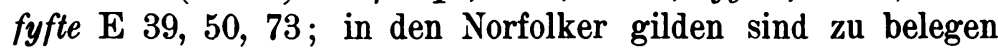
feerde, selten fourte, ferthe, fourthe, twelft (Schultz 33), in Worcester fourth 376. In den Paston Letters erscheinen fourte MP III 134, forte $2 \mathrm{JP}$ III 115, forthe * II 182, twelthe MP II 82 (hauptwort = Twelfth Night!), * II (296).

(§ 232) Von zahlen über 20 werden ordinalia in der Bibel auf verschiedene arten gebildet. 1. Zwei ordnungszahlen werden zusammengefügt (nur in a), z. b. twentithe and firste Hagg. II 2, fourthe and twentithe Dan. X 4, Hagg. II 19, twentithe .. and fourthe Hagg. II 1. 2. Eine oder mehrere ordnungszahlen mit einer oder mehreren kardinalzahlen: three and twentithe 1 Makk. XIII 51, foure and twentithe Sach. I 7, 2 Makk. XI 21, fyue and twentithe Ezech. XL 1 u. ö., hundrid and fouretithe (b: fiftithe) 2 Makk. XIV 4, hundrid and fiftythe 1 Makk VI 20 usw. - fifthe and twenty 1 Makk. IV 52 a, 59 a, seuenthe and twenty Ezech. XXIX 17 a - hundrid ... and eizte and fourtithe 1 Makk. IV 52, 2 Makk. XI 21, 33, hundrid . . and eizte and eiztithe 2 Makk. I 10 - hundred .. and foure and fourtithe 2 Makk. XII 1, hundred and nyne and fourtithe 2 Makk. XIII 1, hundrid ... and seuen and seuentithe 1 Makk. XVI 14. 3. Nur kardinalzahlen werden verwendet: in the hundrid and sixe and fourtie yere 1 Makk. II 70, ebs. hundrid and seuen and fourtye 1 Makk. III 37, hundrid and nyne and fourty 1 Makk. VI 16, hundred ... and oon and fifty 1 Makk. VII 1, hundrid and two and sixti 1 Makk. X $57 \mathrm{a}$, hundred and oon and seuenti 1 Makk. XIII 51. 
(§ 233) Einmal erscheint auch eine einzelne kardinalzahl, wo eine ordinalzahl am platze wäre: in oo zeer of his rewme (b: firste) Dan. IX 1a. Ein ähnlicher fall findet sich bei Pecock: the hundrid parti 15/16. Häufiger ist dieser gebrauch bei Capgrave, vgl. the sevene generacioun 7, the ten zere 166, the elevene zere 243 , the sextene day 232, the fifti zere 230 usw.; die Suffolker testamente schreiben my threty day 1482: 51, die Norfolker gilden y yre der dritte L 58, 65.

\section{v. Fürwort.}

1. Personalpronomina.

(§ 234) Für ae. ic kennt Chaucer noch ich (t. Br. § 250, anm. 1). In den anderen quellen kann ich nur $I, y$ belegen, z. b. Matth. XVIII 18, Pecock 112, Hoccleve MP 2/76 (I : tendrely) u. oft, Lydgate AG 1496, Capgrave Kath. III 1182 (: crye) u. oft, Chr. 1, 2, 4 u. oft. Auch die urkunden und die Paston Letters schreiben durchweg $I$, vgl. Morsbach $120 \mathrm{ff}$., Suffolk 55 u. oft, 1WiP I 19, MP I 257, 1JP I 518, 2JP II 295, 3JP III 12, ebenso Caxton (Römstedt 40). Im Ne. ist ich ausgestorben bis auf Wexford und einen kleinen distrikt im südwesten (Ellis 28, 85).

(§ 235) Für den obliquus pluralis ae. eow tritt im 15. jahrhundert bereits gelegentlich der nominativ ae. $g \bar{e}$ ein, so anscheinend in dem reim sche : * ${ }^{*} e$ (Ms. zow) GR 160, ebs. Chr. 173, umgekehrt gebraucht Capgrave you als nominativ Chr. 2. Hierher scheinen auch einige stellen der Paston Letters zu gehören: Set in sech rewle as ye seme best $1 \mathrm{JP}$ II 209, and ye seme it may do yow good 1JP II 210, I wolle spende $X X d$. or as ye seme to have the sertayn off every thyng her in 2JP III (17), sey for me as ye semyth * I (165), recommaunde me to $X$. Y. ... and all othre suche as ye shal seme gode ${ }^{*}$ III (97), to othere there as ye seme it shulde profite to be knowen F I 398; da dies jedoch die einzigen beispiele sind, ist es möglich, dass hier eine verschmelzung zweier konstruktionen vorliegt: as you semeth, -e (ind. und konj.) + as ye demeth, -e. Wohl ebenso they schall dyspose them ... as they shall seme beste to plese God in dyschargyng ther concyens Suff. 1482: 54.

(§ 236) Statt des ae. akkusativs sing. hine ist überall der dativ him herrschend geworden, doch hat sich hine in den ne. 
südlichen dialekten noch vielfach als $i n, \partial n, n$ erhalten, vgl. Ellis s. 34, 76, 157 u. oft.

(§ 237) Für ae. hēo findet sich überall sche, she, z. b. Matth. IX 18, Pecock 95; für Chaucer vgl. t. Br. § 250, für Gower Fahrenberg 404. Hoccleve reimt shee mit thee MP 26/42 u. ö.,

- Capgrave sche : degre I 992 u. ö., schreibt $s(c) h e$ Chr. 5, 8, 31 u. 0 . Die Londoner urkunden haben neben sche auch (selten) das altertümliche zhe und das nördliche scho (Morsbach $121 \mathrm{ff}$.), die Paston Letters nur sche EP III $278 \mathrm{u}$. oft, 3JP III 43 usw., ebs. Caxton (Römstedt 40). Ellis belegt s. 106 in Hampshire noch $h i<h \bar{e} o$, ferner s. $296 h \bar{o}$ und $\check{s} \bar{o}$ im nördlichen mittelland.

(§ 238) Der - häufig als possessiv verwendete - obliquus sing. fem. lautet gewöhnlich hire; nur selten tritt dafür die sonst im plural gebräuchliche form here ein. Wycliffe gebraucht her nur selten: Gal. IV 25 a, Baruch VI $43 \mathrm{~b}$, Mark. V $23 \mathrm{~b}$, Offenb. XIX $2 \mathrm{~b}$, Pecock schon häufiger (73, 118, $181 \mathrm{u}$. ö.); auch Chaucer reimt here : swere MP 192/421, : gaylere 366/2050, ebs. im Troilus (Kittr. s. 153). Gower hat nur hire (Fahrenberg 391), Hoccleve nur here (: conquere MP 75/70, : were 155/448), ebs. Capgrave (: here < hīeran I 526 u. ö., : bere III 346); doch ist zu beachten, dass es für hire kaum ein bequemes reimwort giebt. In $\mathrm{Chr}$. findet sich denn auch nur zweimal here 57, 160, sonst stets hir 21, 44, $57 \mathrm{u}$. oft. In den Londoner urkunden erscheinen beide formen (in den privaturkunden nur her(e), vgl. Morsbach $121 \mathrm{ff}$., $126 \mathrm{ff}$.), gegen ende

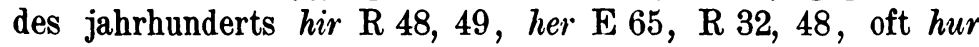
E 65, R 32, 33, in Suffolk hir(e) 1467: 22, 23, 32 u. ö., her(e) 1467: 33, 1470: 45, 1482: 51 u. ö., in Norfolk hire (Schultz 33). In den Paston Letters ist im allgemeinen hir das übliche. Es überwiegt bei MP: hyr I 258, II 25, 26 u. oft, (her ebd. u. ö.), 2JP: III 85, 87, 166 u. ö. - her II (247), III 166, heer (!) III (59) - 3JP: III 32, 43, 69 u. oft (her III 141, 155). Dagegen hat EP her III 278, 279, 280 u. ö., hyr III 27, 1JP her I 275, 276, II 210, hier (!) I (296), 2WiP nur her I 516. Caxton hat wieder beide formen (Römstedt 40).

(§ 239) Der ae. nominativ pluralis hīe ist so gut wie überall durch das an. peir verdrängt worden. he $<$ hie findet sich als seltene ausnahme in den Norfolker gilden (Schultz 33; ebs. in einer Norfolker urkunde von 1426 PL I 17), einmal (MP 
$\Pi$ 216) in den Paston Letters, vielleicht auch bei Lonelich (Ms. they : certeinly XVIII 330), im Ne. in Somerset (Ellis 88). Statt they wird gelegentlich the geschrieben: R 18, MP I 178, 2JP III (162).

(§ 240) Der ae. dativ pluralis him ist als hem noch sehr gebräuchlich, weicht aber allmählich vor dem nördlicheren them zurück, das auf dem ae. demonstrativum $p \bar{e} m$ beruht, während die daneben ebenfalls vorkommende form baym auf an. peim zurückgeht. Wycliffe hat nur hem (Matth. XI 1 u. oft), Pecock dagegen neben hem 2, 3, 8 u. oft schon einmal them 503. Chaucer (t. Br. § 250) kennt nur hem; bei Capgrave ist neben hem (Chr. 1, 3, 6 u. oft) auch dreimaliges them (43, 122) zu belegen. Die Londoner urkunden (Morsbach $122 \mathrm{ff}$.) zeigen ein allmähliches vordringen von them und thaym (daneben selten tham), in den späteren staatsurkunden sind

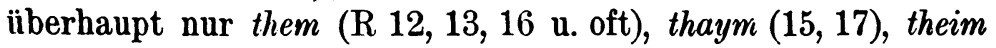
$(11,19,49$ u. o.), thaim $(43,45,46)$ zu finden. Die Suffolker testamente kennen sowohl die alten wie die neuen formen: hem 1467: 17, 27, 38 u. ö., them 16, 17, 1482: 60, theyn 1481: 66,67 ; die Norfolker gilden haben einmal yam, sonst hem (Schultz 33), die späteren urkunden derselben grafschaft nur them 1482 III 286, 287 und thaym 1459 I 461, in Worcester findet sich seltener hem 383, 386, 398 als them 376, 377, 379 u. oft, theym 401, 408. In den Paston Letters herrschen grosse verschiedenheiten. Wo hat nur hem I 404, II 174, ebs. meist Fa (I 171, 172, 314 u. ö.; doch auch theym I 183, 314, 321), 1JP und MP etwa gleich häufig hem I 348, II 210; I 111, 179, II 240 u. ö. und them I 349, 518 - auch hym MP I 110, II 178 - ; I 110, II 240, III 135, EP und 2JP nur them III 252, 279, 280; III 22, 85, 199 u. ö., 3JP hem III 57, them III 7, 20, theym III 20,48, 49 u. ö. Caxton hat meist them, daneben auch theim und hem (Römstedt 40), RF 114 erscheint them einmal für den nominativ. Auch im reinen süden erscheint thaym im 15. jahrhundert, z. b. in einer Kenter urkunde von 1448 (LC 197). - Die ne. mundarten bieten häufig $\mathrm{em}$, das doch wohl auf hem beruhen wird, wenn auch anlautendes $p$ bisweilen verstummt, so in pis, pan, poet in Somerset und Devonshire (Ellis 150, 163). 


\section{Possessiva.}

(§ 241) Die possessivpronomina der 1. und 2., sowie das maskulinum sing. der 3 . person bieten nichts bemerkenswertes. Für das femininum sing. der 3. person wird der genetiv here, hire verwendet, vgl. $\S 238$.

(§ 242) Für den plural der 3. person wird gewöhnlich ebenfalls der genetiv here, hire gebraucht, und zwar ist here bei weitem das üblichere; doch kommt bereits ein auf an. peira beruhendes selbständiges possessivpronomen auf. Wycliffe hat meist her(e), selten in der älteren fassung hir Dan. III 94, Luk. XXI 12, 2 Kor. XI 15, doch auch there Matth. III 6 a, $\mathrm{X} 17 \mathrm{a}$, theire VI $7 \mathrm{a}$, VII $6 \mathrm{a}$ - auffällig ist the kingdam in heucnes is heren, herun ( $\mathrm{b}$ : herne) Matth. V 3, 10 -, Pecock einmal hir 454, sonst her 3,5,9 u. oft, Chaucer dieselben beiden formen (t. Br. § 251), Capgrave stets her(e) 12, 28, 35 u. ö. In den Londoner urkunden findet sich here, aber auch theire, thaire, there, das im laufe der zeit zunimmt (Morsbach 127); gegen ende des jahrhunderts sind überhaupt nur their

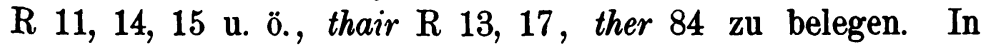
Suffolk erscheinen gleich häufig here 1467: 17, 21, 30 u. ö. und ther(e) 1467: 19, 30, $43 \mathrm{u}$. 0 . In den Norfolker gilden ist ther noch selten (Schultz 34), häufiger in den späteren urkunden derselben gegend (ther, their 1482 III 285, 288, 289, her(e) 1451 I 189 u. ö., 1482 III 288); in Worcester ist hur 377, 378,380 u. ö. gebräuchlicher als their 376 , ther 378,380 u. ö. In den Paston Letters hat Wo her I 404, theyr I 404, II 174, MP etwa gleich häufig her(e) I 81, 109, 110 u. ö. und ther(e) I 178, 202, II 178 u. ö.; die übrigen schreiber verwenden nur die an. form: Fa their u. ä. I 172, 174, 175, ther I 314, 1JP ther I 348, 2JP ther III 164, 166, 222, ebs. 3JP III 32, 40, 50. Auch bei Caxton ist their die regel (Römstedt 41).

\section{Demonstrativa.}

(§ 243) Von dem einfachen ae. hinzeigenden fürwort ist überall der nom. neutr. paet als demonstrativpronomen erhalten. Der dativ $\bar{p} \bar{c} m, \not \bar{a} m$ wird als plural des personalpronomens verwendet (\$ 240), nur ganz selten im singular als demonstrativum: at pen enteryng Norf. gilden 41, for than Pecock 345, bi than that MP III 126, for thon Caxton RF 28, 36; pāere 
kann ich nur aus den Norfolker gilden belegen (yer-qwile BL 72). Der plural $b \bar{a}$ dagegen ist als tho(o) noch ziemlich gebräuchlich, so bei Wycliffe Matth. XIII 17, Luk. I 39, Dan. II 44, III 94, I 2 b, II 43 b, Matth. III 1, Offenb. IV 9 u. ö., Pecock (1, 2, 3 u. oft), Chaucer (t. Br. \$252), Capgrave (2, 3, 8 u. oft), den urkunden von London (Morsbach $128 \mathrm{f}$; später nur E 69), Suffolk (1467 : 16, 25, 29 u. ö.) und Norfolk (Schultz 34); seltener ist es in den Paston Letters: F I 175, MP I (438), II 196, (385) u. ö., Norf. I (89), * (214) und bei Caxton (Römstedt 41).

(§244) Gebräuchlicher ist ae. pes, peos, pis. Für den singular wird allgemein das neutrum this verwendet, nur selten das maskulinum: yes $\mathrm{N} 30$, thes MP I 202, II 249, III 24, 1JP I 234, II 210. Für den plural sind fast allein die aus dem singular gebildeten formen thise und these gebräuchlich. Wycliffe hat thes(e), vgl. Gasner 83, ebs. Pecock 8, 13, 20 u. ö., doch auch this 496, Chaucer beide formen (t. Br. 252), Capgrave this 156, sonst stets these 6, 12, $15 \mathrm{u}$. oft. In London sind beide wörter üblich (Morsbach $128 \mathrm{f}$.), ebenso gegen ende des jahrhunderts. Auch in Suffolk herrschen ausschliesslich thes 1467: $42 \mathrm{u}$. oft, thees 1481: 64, theyse 1482: 54, in Norfolk wieder beide formen (Schultz 34), ebenso in den Paston Letters und bei Caxton (Römstedt 41). Selten ist ne. those (thoos, thos); ich finde es nur in den jüngeren staatsurkunden ( $\mathrm{R} 68$, 70, 72 u. o.), in den Paston Letters (3WiP +III 344) und bei Caxton (Römstedt 41).

(§245) ae. ilca erscheint als ilk, thilk noch bei Wycliffe (Gasner 83), Pecock (3, 8, 15 u. oft), Chaucer (t. Br. § 255) und in den Norfolker gilden (Schultz 35). Im Ne. ist es in manchen gegenden des südwestens als $\succsim i k$, $\delta \alpha k$ sehr gebräuchlich, vgl. Ellis 44, 104, 160.

(§ 246) ae. self, sylf erscheint auch im Me. in diesen beiden formen. Die Bibel hat $e$ und $i$ (Gasner 81), Pecock ausser self 230 nur silf $3,13,15 \mathrm{u}$. oft, Chaucer nur $e$ (t. Br. § 255), ebenso Capgrave $(75,90,184$ u. ö.) und die urkunden von London (Morsbach 129; R 13, 33, 77 u. ö.), Suffolk (1467: 15, 22, 25 u. ö.) und Norfolk (Schultz 35; PL 1451 I 189, 1459 I 461 u. ö.). Dagegen herrschen doppelformen in den Paston Letters. Fa hat self I 321, silf I 398, Wo sylf I 370, 374, 2 WiP silf III 113, self(f) I (302, 303, 493), MP sylf III 194, sonst self I 111, II 26, 212 u. ö., 1JP self II 218, 221, 2JP 
sylfe u. ä. III 114, 188, II (322), selff(e) III 116, 187 f. u. ö., $3 J P$ self II $(357,400)$, sonst stets $\operatorname{sylf}(f)$ III 43, 73, 110 u. ö. Caxton hat nur $e$ (Römstedt 42).

\section{Relativa und Interrogativa.}

(§ 247) ae. $h w \bar{a}$ ist als who in allen denkmälern häufig. Einmal wird in der art des 16. jahrhunderts der obliquus hom für den nominativ gebraucht Norf. ${ }^{+}$PL III 337 , umgekehrt ho als akk. MP I 112.

(§ 248) Neben ae. hwelc existierte auch eine form hwilc, hwylc mit einer anderen ablautstufe des stammes hwa. Beide haben sich als whech, which im südlicheren England erhalten. Die Oxforder und Chaucer kennen nur which(e), vgl. Gasner 84, Pecock 2, 3, 5 u. oft, t. Br. $\S 254$, dagegen bevorzugt Capgrave whech $2,3,4$ u. oft bei weitem (which 1, 15, 37 u. ö.). Die Londoner urkunden zeigen doppelformen; doch kennen schon die staats- und parlamentsurkunden nur which (Morsbach $64 \mathrm{ff}$.), und auch gegen ende des jahrhunderts ist es allein gebräuchlich (R 13, 14, 32 u. ö.). In Suffolk kommt neben wiche 1467: 19, 20, 21 u. ö. auch $w(h)$ eche 1481: 58, 1482: 52, 54 vor. Norfolk verwendet 1389 nur which (e), daneben qwyche, aber auch nördliches qwilk (Schultz $34 \mathrm{f}$.), in den späteren urkunden ist neben which $(e) 1459$ I 461, 1482 III 282 u. ö., qwiche 1465 II 224, anch wheche 1451 I $190 \mathrm{zu}$ belegen. In Worcester erscheint which 377,386 . In den Paston Letters herrscht starkes schwanken. Nur which u. ä. (über den anlaut vgl. § 213) haben Wo I 370, 433, EP III 279, 2JP III 85, 117, 187 u. ö., meist ebs. 3JP III 48, 49, 50 - wheche II (303). Dagegen hat F öfters weche I 182, whech I 93, 94 usw. neben häufigerem whiche u. ä. I 93, 174, 175 u. ö., ebs. MP wech(e), whech II 216, 217, 240, whesch III 24, qheche I 82, 110 neben häufigem $w(h) y$ che I 537, 538, II 82 u. ö., 1JP schreibt öfter whech I 209, 232, 234 u. ö. als which I 232, 252, 275, $1 \mathrm{WiP}$ nur wheche I 30 , 2WiP nur qweche I 86, 516, II 109 u. ö. Für Caxton erwähnt Römstedt s. 42 nur whiche.

(§ 249) Das korrelativum zu hwelc lautet ae. lautgesetzlich swelc, mit angleichung an hwilc auch swilc. Im Me. finden wir daher zunächst swech und swich, mit ausfall des $w$ auch sech und sich, daneben die verdumpfte form such. Wycliffe hat siche und suche (Gasner 109), ausnahmsweise auch seche 
2 Makk. VIII 7 b, Pecock nur such 2, 3, 7 u. oft, Chaucer swich und such (t. Br. § 255), Capgrave stets swech 108,110, 123 u. ö. In den Londoner privaturkunden ist $\operatorname{such}(e)$ allein gebräuchlich, in den staats- und parlamentsurkunden erscheinen daneben auch swiche und $\operatorname{sych}(e)$, die jedoch am ende des jahrhunderts wieder verschwunden sind (nur suche $\mathrm{R} 11,12,13 \mathrm{u}$. ö.). In Suffolk ist am häufigsten suych 1467: 20, 22, 25 u. ö., seltener such 19, 28, 30 u. ö., einmal sweche 1481: 56. Die Norfolker gilden schreiben swilk, swiche, einmal soche (Schultz 35), die späteren urkunden derselben gegend seche 1451 I 189, 191, aber meist such 1459 I 461, 1482 III 286 u. ö., in Worcester findet sich $\operatorname{such}(e) 378,379,380$. In den Paston Letters herrscht wieder grosse mannigfaltigkeit. Nur $\operatorname{such}(e)$ erscheint bei Fa I 172, 174, 175 u. ö., Wo I 370, 371, 400 u. ö., 2WiP II 109, III 259, EP III 251. 1WiP schreibt swich I 26, MP swyche I 177, 178, 179 u. oft, syche II 82,83 , seche I 111, 112, 113, soche I 111, III 29 u. ö., such $(e)$ II 185, 195, 240 u. ö., 1JP suyche I 518, such I 233, II 220, 221, $\operatorname{sech}(e)$ I 348, II 209, 210 u. ö., 2 JP syche II $(245,321)$, suche IIJ $8 \dot{6}, 100,101$ u. ö., 3JP suche III 56, syche III 40, 43, $48 \mathrm{u}$. oft. Caxton hat suche $4,5,6$ u. oft.

\section{Verbum.}

\section{Bildungsweise der starken verba (ablautreihen).}

$$
e i \text {-reihe (klasse I). }
$$

$(\S 250)$ In der älteren fassung der Bibel sind die ae. ablautverhältnisse noch streng gewahrt. So lautet das pt. pl. abiden 1 Petr. III 20 u. ö., dryuen Hebr. XI 34 u. ö., risen 1 Makk. IX 40 u. ö., smyten Apg. XVIII 17 u. ö., wryten 1 Makk. VIII 22 u. ö., witen 1 Thess. IV 2 u. ö. In der jüngeren fassung dagegen ist die dritte ablautstufe bereits einige male der zweiten angeglichen worden; so heisst es aboden Apg. XX 5, smoot Matth. XXVII 30, smoten Apg. XVIII 17, wroten Apg. XVIII 27 neben dryuen Apg. XIII 50 u. ö., rysen Apg. VI 9 u. ö., smyten Luk. XXII 64 u. ö., writhen Joh. XIX 2, witen 1 Thess. IV 2 u. o. Ein abweichendes pt. sg. hat in beiden fassungen ae. stīgan entwickelt: steiz Matth. III 16 a, stiez (b: stiz) 1 Makk. XIII 2; diese im süden häufige form wird wohl nicht als übertritt in die $c u$-reihe, die schwächste 
von allen, aufzufassen sein, sondern als angleichung an die reduplizierten verben (vgl. § 251, 251 apt. smet, bleef, grepe zu smītan usw.). Gewöhnlich ist stīgan indessen schwach geworden, vgl. stiede Ezech. XI 23, XL 6, steyede ebd. 31 a, stieden Ezech. XL 49 u. ö., stizeden Apg. XXI 15 a, Offenb. XI 12 a (b: stieden), steiziden Ezech. XXXVI 3 a, XXXVII 8a u. ö. Ebenso finden sich zu smitan die schwachen formen smytidist Ezech. XXV 6, smytiden 1 Makk. II $44 \mathrm{~b}$, VIII $4 \mathrm{~b}, 2$ Makk. I $16 \mathrm{~b}$, zu scinan shynyde 1 Makk. VI 39, 2 Makk. I 32, X 35, shynyden 1 Makk. VI 39 (pt. sg. shoon(e) Ezech. XLIII 2a, 2 Makk. I 22). In b werden noch andere verba schwach abgewandelt, vgl. abididen Ezech. X 16, 1 Petr. III 20, dryueden 1 Makk. I 56, Hebr. XI 34, risiden Dan. XIII 19, 61, 1 Makk. IV 52, IX 40 u. ö., writiden 1 Makk. VIII 22, XIV 18, 26. - Zu frz. estriver findet sich das pt. stroof (b: stryuede) Ezech. XX 36 a.

(§250 a) Bei Pecock ist ausgleich überall eingetreten, vgl. abode $(n)$ 19, 206, ro(o)sen 494, 497, wroten 21, 22, 31 u. ö.; schwach ist stied 60 . Chaucer hat die ablautsverhältnisse durchweg bewahrt (t. Br. § 153); dagegen nähern sich einige seiner schüler bereits stark dem ne. standpunkt. Lydgate reimt abode : stode AA III 93, aroos : purpoos EFr III 706, roode : he bestroode AG 799 u. ö.; ferner smette pt. : mette (traf) Th $366 \mathrm{c}$. Im Generides findet sich aboode : he rode 4028, : broderhode 6253, in Part. glode (zu glidan) : brode 726. Zu erwähnen sind ferner sty : truly bei Hoccleve (Vollmer 220), smet pt. sg. : let (hindernis) Bokenam I 622. Aus der volkstümlichen litteratur führe ich an abood : vndirstood Lon. XX 156, : knyghthoode Ryman CXLIII 4, rode : brode < brād Beryn 1911, stide (zu stīgan) : side Ryman XLIV 9, steyde : seide : leyde ebd. XLVI 7, XLVIII 6, ryste : Cryst Rom. Auf. 81.

(§ 251) Capgrave hat meist abydyn u. ä. 208, 218, 302, ridyn u. ä. 171, 235, 271 u. ö., risin u. ä. $69,85,108$ u. ö., wrytyn u. ä. 70,116, 172 u. ö., aber auch abode 168, 194, rood 248, wrote 171, ferner steye (: hey) Kath. I 393, smet pt. sg. 34, 45, 88 u. ö., pt. pl. 109, 159, 237 u. ö.

In den urkunden fehlen belege so gut wie ganz; in London findet sich writen (Morsbach 138), in Norfolk rised (pt. sg.) BL 81, 96.

In den Paston Letters sind die jüngeren formen das gewöhnliche, vgl. abode Norf. II 55, drove MP II 196, roode 
u. ä. MP II 99, 240, Norf. II 59, wote u. ä. Wo I 370, MP II 83 u. ö., 2JP III 100, 115 u. ö., wrote u. ä. Wo I 404, MP II 179, 195 u. ö., 2JP III 101, seltener sind abedyn MP I 111, Norf. I 199, dreave 3JP III (53), reden u. ä. MP I (224, 269), wet MP III 45 , wrete $*$ II (38). Ausgleichung des singulars nach dem plural und infinitiv kann eingetreten sein in wett AP I (89), we(e)t 2JP III (60), 3JP III 41 (doch vgl. § 47); schwach ist wrytted AP I (88), wohl ebs. stede (statt stīde, vgl. $\S 104)$ Norf. II (205); auffällig ist weit 3JP III 68 (an.?, vgl. auch $\S 47)$.

(§251 a) Bei Caxton ist meist ausgleich eingetreten, so in $\bar{a} b \bar{d} d a n$, ārīsan, smītan, witan (vgl. Römstedt 42, 47), dazu bote RF 91, strof (zu estriver) 83; erhalten ist die ae. ablautform nur in ryden 39 , smyten 86 , smeton 27 . Nach der reduplizierenden klasse scheinen gebildet sein pt. sg. bleef (ae. belīfan) $\mathrm{RF} 15$, grepe (ae. grīpan) 111. $\mathrm{Zu}$ ae. strīcan kommen nur schwache formen vor, gelegentlich auch $\mathrm{zu}$ rīdan (vgl. Römstedt 42) und wīcan RF 67.

$$
\text { eu-reihe (klasse II). }
$$

(§ 252) Bei Wycliffe erscheint ausgleich des prät. pl. nach dem sing. nur in einem falle: chesen Luk. XIV $7 \mathrm{~b}$ nach dem sing. chees u. ä. 1 Makk. III 38, IV 35, Mark. XIII 20 u. ö. (dazu die schwache form chesiden Luk. XIV 7 a, 1 Makk. I 65). Doch sind starke formen nach dieser klasse nicht sehr häufig. Von beodan sind erhalten das pt. sg. forbeed Matth. III 14, Amos IV $7 \mathrm{~b}$, Ezech. XXXI $15 \mathrm{~b}$, forbede 2 Petr. II 16 , forbedde Ezech. XXXI 15 a und das pz. (for)boden u. ä. 1 Makk. XIII 49, II 64 b, Mark. IX 37 b, Luk. XIV 8 b usw., Luk. IX 49 a, XI 52 a, Röm. I 13 a, XV 22 a, Apg. XVI 6 a. Zu flēogan erscheint ein regelmässiges pt. sg. fleiz $(=$ floh $)$ Mark. XIV 52, Apg. XX 27 (b: fley), 1 Makk. I 19 (b: flei) u. ö., ebs. flyz Apg. VII 29 (b: flei), ferner (= flog) fleez (b: fley) Hosea IX 11 u. ö.; das pt. pl. lautet flowen Luk. VIII $34 \mathrm{~b}$ (flohen), Offenb. XIX 17 b (flogen), daneben flewen (red.?) ebd. a. Meist ist das pt. pl. nach an. flöja schwach gebildet, vgl. fledden Obadja 14, Nahum II 8, Sach. XIV 5 u. ö., wozu in b auch ein pt. sg. fledde Ezech. XXXIII 22, Dan. II 1, Hos. XII 12 u. ö. erscheint. Nur schwach sind die wenigen belege für ae. bügan, lēogan und lēosan, vgl. bowide (pt. sg.) 1 Makk. V 68, 
bowid (pz.) Habak. III 6, leezide, leizide (pt. sg., b: leizede, liede) Dan. XIV 11, 18, 1 Makk. XI 53, XIII 19, lostist (pt. sg.) Ezech. XXVIII 17. Zu ae. sēoðan erscheint in a das pz. sothen Dan. XIV 32 b, Ezech. XXIV 5, 10 mit aufhebung des grammatischen wechsels, in b soden, sodun a. a. 0 .

Pecock bildet zu bēodan das pt. sg. bede 322, zu cēosan das pt. sg. chase 278, 349, 430, einmal chose 135 (unsicher 168) mit der westlichen angleichung des prät. plur. an das pz.; das pt. pl. lautet chosen $448 \mathrm{u}$. wechsels. lēosan ist schwach geworden (pz. lost 182, 307).

(§ 252a) Chaucer bewahrt im allgemeinen wieder die ae. ablautsverhältnisse; jedoch ist der vokal des pz. stets in den pl. pt. und in einem falle (shoot) auch in den sing. gedrungen; der grammatische wechsel ist bei sēoðan erhalten, bei cēosan aufgegeben. Ganz oder teilweise schwach geworden sind clēofan, créopan, flēogan, lèosan (t. Br. $§ 156 \mathrm{ff}$.). Hoccleve hat westlichen ausgleich in sook (: strook sbst. RPr 1326), übertritt in die $\breve{e}$-reihe im pt. sg. claf (zu clēofan) : staf MP 172;914, Lydgate bietet das pt.sg. chees (: prees gedränge $\mathrm{ScPh} 614$, ebs.

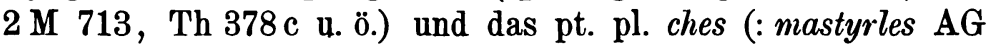
882). Aus der volkstümlichen litteratur sind bemerkenswert ches (2. sing. prät.) : pes : ces : reles Brompton 36, lest (pz. zu lèosan) : beheest Beryn 1509 (neben häufigerem lost), ebs. (Ms. lost) : blest : best Audelay 54, ebs. 17, 42.

(§ 253) Bei Capgrave lautet von ceesan das pt.sg. chese 132 chase 60, 132, das pt. pl. chose 20,62, 132 u. ö. und - in Kath. - chees (: douteles) III 860, das pz.chose (: rose : glose) Kath. III 947, chose(n) Chr. 20, 50, 208 u. ö. Zu fléogan erscheint das pt. sg. fley 139 (flog); in der bedeutung 'fliehen' ist an. flöja herrschend geworden, vgl. pt. $f l e d(d e) 79,91,98$ u. ö. $\mathrm{Zu}$ beodan kommt nur noch vor das pz. forbode 205, 222, die übrigen formen werden durch biddan ersetzt. lēosan ist stets schwach: pt. sg. lost 69, 75, 119 u. ö., pz. lost 279.

(§253a) Aus den urkunden sind nur zu erwähnen das pt. pl. chosen Morsbach 138, pz. lost Morsbach 140, R 19, 83, pz. loken Worcester 379.

In den Paston Letters ist belegt $\mathrm{zu}$ beodan das $\mathrm{pz}$. bodyn 3JP III 31 (pt. pl. bade usw. s. § 265), zu cēosan das pt. sg. chase 2JP II 295, zu lēosan lost (pt. sg.) 3JP III 220, 
(pt. pl.) 2JP III 130, (pz.) 2JP III 101 u. ö., zu scēotan das prät. plur. schotte Devon I (85), * I (427) und der analogische sing. shott 2JP III 104, ähnlich zu scüfan das pt. sg. schoffe AP I (217). 'Sie flohen' heisst fled(d)e MP II 240, 2JP III (162). Ae. crüdan ist erhalten im pz. crod MgP III (215).

Bei Caxton ist der vokal des partizips überall in das prät. sing. gedrungen; eine beträchtliche anzahl von verben

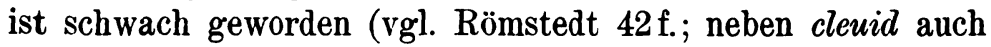
das pz. clouen 63).

\section{$\breve{e}$-reihe: verba mit gedeckter liquida oder nasalis (klasse III).}

(§ 254) Bei Wycliffe entspricht das pt. sg. meist unmittelbar der ae. form, z. b. bond (b: boond) Mark. VI 17, Luk. X 34, fonde, foond(e) 1 Makk. VI 63, Matth. VIII 10, XVIII 28, Ezech. XXII 30 u. ö., fauzt(e) Sach. XIV 3, 1 Makk. V 50, VI 63 u. ö. Auf einer ae. analogisch gelängten form gea ald wird beruhen ze(e)lde Ezech. XXII $31 \mathrm{a}, 1 \mathrm{Tim}$. VI $13 \mathrm{a}$. Westlicher ausgleich ist eingetreten in bound Matth. XIV 3, vnbound Apg. XXII 30, found Hebr. XII 17 a, sprung(e) Matth. XIII 6 a, Luk. VIII $6 \mathrm{~b}$, fouzte 1 Makk. X 75 b. Im prät. pl. herrscht überall derselbe vokal wie im partizip (z. b. holpen Sach. I 15a); der unterschied gegenüber dem singularvokal ist meist erhalten, z. b. in bigunnyn Mark. II 23, V 17, bounden Joh. XVIII 12, XIX 40 u. ö., drunken u. ä. Ezech. XXXIV 18, 19, Mark. XIV 23, Offenb. XVIII 3 u. ö., fouzten 1 Makk. III 2, VI 31 u. oft, founden Matth. II 11, Mark. XI 4 u. ö., zoldun Apg. IV 33 a, runnen Mark. VI 33, Joh. XX 4, Apg. XXVIII 15a, sungen 1 Makk. IV 24, Offenb. V 9, kunne(n) Luk. XI 13, XII 56 b; auch songen 1 Makk. IV 13? Ausgleich nach dem pt. sg. zeigt die ältere fassung in biganst Dan. II 29, brasten 2 Makk. XIV 43, fauzten Offenb. XII 7, foonden 2 Makk. I 20, ze(e)lden 1 Makk. X 41, Apg. XVI 2, swame 1 Makk. IX 48, die jüngere nur in brasten 2 Makk. XIV 43, fonden Luk. XIX 32, zeelden 1 Makk. X 41, ranne Mark. VI 55, Apg. VI 12 . Zu ae. ceorfan, gieldan und helpan erscheinen auch schwache formen: keruyden 1 Makk. I $59 \mathrm{~b}$ (doch pt. sg. karf Amos I 13 und pz. coruen Hos. XIV 1, Sach. XIV 4), zeldide Nahum II 2, 1 Makk. IX 72, X 9 u. oft, helpiden Sach. I 15 b. Das pt. prs. ae. dearr erscheint als doren (pl.) 2 Kor. X 12.

Anglia. N. F. XII. 
Pecock (belege spärlich) schreibt im pt. sg. biganne 24, 250, im pt. pl. einerseits founden 14, 18, 243 u. ö., sungen 205, kunnen 2, 123, 213 u. oft, andrerseits biganne 41, fonden 242.

(§ 255) Bei Chaucer ist überall das prät. pl. dem pz. angeglichen worden; auch dient das pt.sg. bisweilen als plural (t. Br. $\S 138,193$ ); ähnlich sind die verhältnisse bei Gower (Fahrenberg 406). Bei den Chaucerschülern ist der nördliche ausgleich schon häufiger; Hoccleve hat dranke und can neben konne (Vollmer 220), Lydgate began : man AA I 747, ranne : man AA II 1521 u. ö. (doch s. a. § 36), aber auch begunne (wie statt began $\mathrm{zu}$ lesen ist) : sonne AA II 1710, ronne : sonne < sunne Th 371 a, conne : sponne pz. : sonne MP 260/488 u. ö., Bokenam fonde : londe VIII 1152, FL braste : stedfaste 490, began : manne : ranne (pl.) 282 , Gen. wanne : ranne (sg.) 4974, Wade rane : mane : thane 1906, 1942; auf ausgleichung nach dem pz. deuten die reime founde (pt. sg.) : bounde (pz.) Lydgate AA III 903, : confounde usw. Bokenam VIII 372, : grounde : round CL 221, : ground Gen. 3930, : stounde ebd. 5661, Part. 701; an Wycliffes zeldide erinnert zeld (pz.) : felde Pall. 207/17. Aus der volkstümlichen litteratur belege ich blane (pt. pl. zu blinnan) : came GR 388, fond (pt. pl.) : hond Lon. XXVI 400, auch can, con (= cunne) : sunne Audelay 46, Abr. Is. 100.

(§ 256) Bei Capgrave ist das pt. sg. meist regelmässig : bond 269, faute 155, 215, 239 u. ö., fond 2, 96, 97 u. ö., zald 271, malt, ran 9; einmal erscheint found 86 mit dem partizipialvokal. Im plural sind neben begun(ne) 7, 161, 197, founde 126, 202, 209 u. ö., foutyn 47, zold(e) 187, 188, 189, holpe 182, runne 69 (trans.!), 152 und sprong 83, wonne 136 auch began 67, 143, bonde 126, faute 156, 276, 295 u. ö., fond 92, 93, 113 u. ö., can 50, dare 23 anzutreffen. Kath. bietet an reimen nur we can : man Pr. 214 u. ö.

(§ 256 a) Fast keine belege bieten wieder die urkunden. Morsbach (s. 141, 148) zitiert pl. konne, dur, fond, Schultz (s.40) begunne(n) und pz. hulpen (!); hinzuzufügen ist can (pl.) R 19, holpe (pt. pl.) Suff. 1467: 28, founde (pt. sg.!) BL 83.

In den Paston Letters sind die belege spärlich und selten gut überliefert. Nur zu findan sind ausreichende beispiele vorhanden : pt. sg. fond $u$. ä. MP I 179, II 185, 2JP III 222 u. ö., 3JP II (343), fend (!) MP I 111 (vgl. § 47 ?), 
daneben in westlicher art fund(e) 2WiP I (493), 516 u. ö., fownde MP II 216, 217, 2JP III 129, 160. Der plural ist nur einmal * II 404 als fond belegt; bemerkenswert ist pz. fond (vgl. § 124) 3JP III 256, 257. Ferner erscheinen pt. sg. holp F I (170) - westlicher ausgleich - pt. pl. bygonne Norf. I (211), ronne * I (74), faothe Devon I (85), fawzthe Suff. I (429), can u. ä., MP II 187, 1JP I 211, 2JP III 86 und stets, dor < durron MgP III (215), ther MP II 187, 195, (226). Zu gieldan gehören die auffälligen pz. yeldyn $2 \mathrm{WiP}$ (?) II (6), yelden * II (111), yolded Devon I (85), die durch vermischung starker und schwacher formen entstanden sind.

(§ 256 b) Bei Caxton ist das pt. sg. mit westlichem ausgleich gebildet in broste (ae. berstan), fought - vgl. Römstedt 43 neben faught RF 107 und in founde 34 neben häufigem fonde 7 u. oft. Die dritte ablautstufe ist nur noch erhalten in begonne 11, worden 34 und conne 60,62, 65 u. ö. - auch ronne 87 ? vgl. sg. ronne 20 neben häufigem $\operatorname{ran}(n e)-$, sonst ist überall nördlicher ausgleich eingetreten, vgl. bonde 116 , fonde 48,55 , 60 u. ö., began 35, helpe 101 (zum pt. sg. helpe 69, 78), ranne 15, 17, 22 u. ö., sprang(e) 27, 97, can 25, 30, 38 u. ö., dar 64, 72; daluyst 41. Schwache formen erscheinen zu ae. brennan (stets), helpan, slingan (Römstedt 43).

$\breve{e}$-reihe, verba mit einfacher liquida oder nasalis (klasse IV).

(§ 257) Bei Wycliffe sind nur drei verba ausreichend belegt, ae. brecan, beran und cuman, dazu das pt. prs. sculan. Von brecan lautet das prät. sing. brak Matth. XIV 19 u. ö., das prät. plur. nicht * breken, sondern braken Daniel IX 11, Hosea VI 7, VIII 1, 1 Makk. XIII 44 u. o. mit ausgleichung nach dem singular. Die jüngere fassung bietet einmal (Micha III 3) broken mit dem vokal des partizips. Von beran erscheint ein regelmässiges prät. sing. bar Joh. I 32, Apg. XV 8, 1 Petr. II 24 u. ö. und mit längung des stammvokals baar, bare Matth. XIV 11 a, Mark. X 14 u. ö., daneben einmal bere Matth. VIII 17 a. Selir selten hat die dritte ablautstufe das regelrechte $e:$ pt. pl. beren Luk. V $18 \mathrm{~b}$, Hebr. XII $20 \mathrm{~b}$. Gewöhnlich ist auch hier der singularvokal massgebend gewesen, vgl. baren Luk. VII 14, Apg. V 10, Hebr. XII 20 a, Mark. XIV 57 b, Joh. II 8 b. Für cuman wären com und comen zu erwarten, doch findet sich nur comen 
Mark. III 20 a, 31 a und thou come Joh. VI 25 b. Die gewöhnliche pluralform ist camen (z. b. Mark. III 22, Off. VII 14, Ezech. IX 2), eine angleichung an den allein üblichen neugebildeten sing. $\operatorname{cam}(e)$ Matth. III 1, V 17, Off. VIII 3 u. oft. Der plural von sculan lautet meist shulen Matth. XXI 22, 31, seltener schal Matth. XII 41 a, XXI 2 a, Mark. XIII 25 a, 1 Petr. IV 18 a, Dan. XI 30 a; ausnahmsweise wird shul auch als singular gebraucht, so Ezech. XII 12 a.

Die bei Wycliffe gewöhnlichen formen werden bei Pecock ausschliesslich verwendet, vgl. pt. sg. bare $300 \mathrm{u}$. ö., 2 pers. barist 200, 262, pt. pl. bare(n) 205, 530, pt. sg. brake 147 u. ö., pt. sg. came 18, 56, 102 u. oft, 2. pers. camest 205, pt. pl. camen 18, 247, 278 u. ö., pt. sg. stale 155. Der pl. zu sculan lautet gewöhnlich schulen (43 u. oft), einmal schal 26.

(§257 a) Bei Chaucer sind im gebrauch coom und cam, coomen und camen, noom und nam; das pt. sg. von beran lautet bar und beer; angleichung des pl. pt. an den sg. pt. scheint nur in shal neben shullen eingetreten zu sein (t. Br. $§ 142,193,198)$. Hoccleve bildet zu beran die pt. sg. baar und beer, zu cuman und niman die pt. sg. cam, nam (Vollmer 219 f.); Lydgate bietet cam pl. (: than AG 862 u. ö.), bare sg. (: care AG 807, ebs. AA I 644), bar pl. (: squar EFr III 1449) und bore pl. (: sore AG 1895), Bokenam cam pt. sg. (: insam I 967), come pt. sg. (: Rome IV 238), shal pl. (: especyal VIII 276, ebs. XII 274), in FIL erscheint bere für pt. sg. und pl. (: baneve 213, : saphere 223), in Gen. ebenfalls bere pt. sg. (Ms. bare : banere 2215), ferner brake pt. pl. (: make 6085), in Part. came pt. sg. (: shame 2843, ebs. 2661, 3432), tere pt. sg. (: bere < bera 5893), bei K. Orl. bore (pt. sg.) Münster 6. Aus der volkstümlichen litteratur sind bemerkenswert cam pl. sg. : than Lon. XXXIV 325, ebs. XVIII 202, come sg. : some < suma XXX 439, cam pt. sg. : game Beryn 1005, ebs. 1273, come pt. sg. : anoon 2268 und mit ausgleich nach dem pz. to-brook pt. pl. : ontook Lon. XVIII 321, bare : before Audelay 20, torne pt. pl. : scorne : thorne Rym. XLVI 5; eine schwache form scheint vorzuliegen in berid (ae. bēeron) : deied : aspied : cried Lamb. II 48/218.

(§ 258) Bei Capgrave sind zu belegen beran, brecan, cuman, niman, stelan, sculan. $\mathrm{Zu}$ ersterem lautet das pt. sg. nur bar 10, 201 und bare 5, 33, 258 u. ö., ebs. in Kath. (: bare adj. : declare III 968), der plural in Kath. bere (: here Pr. 151, ebs. 
III 967), in Chr. bare 101, 265 und bore 175, 207 mit verschiedenartigem ausgleich. brecan erscheint im pt. sg. als brak 243, 264, 294 u. ö., brake 251, jedoch auch als brok 235 mit dem partizipialvokal, nur so belegt ist das pt. pl. (broke 129, 143,316 u. ö.). cuman lautet im pt. sg. come (: dome Kath. I 315, ebs. I 703; Chr. nur 18) und cam (: am < eam Pr. 241, Chr. 17, 18, 61 u. oft), im pt. pl. come (: crystendome I 444, Chr. 17, 18, 20 u. ö.) und häufiger cam, came (: wan pt. sg. zu winnan II 1301, Chr. 12, 18, 222 u. ö., camme 36). Zu niman ist belegt das pt. sg. nam (: woman IV 1692), zu stelan pt. sg. stal 173, pt. pl. stole 316 . Der plural von sculan lautet in Kath. nur schall (: all : fall II 1327; zu -ul kaum reimwörter), in Chr. shul 12, 17, 20 u. ö. und schal 14, 95, 149 u. ö. Eine singularform schul erscheint $51,80,85$ u. ö., jedoch fast stets an stellen, wo das prät. schuld zu erwarten wäre (s. §179, doch auch $\S 257,259,259$ a).

(§ 259) Die urkunden bieten wenigstens einige belege. Morsbach zitiert $(\mathrm{s.138,148)}$ kom pt. sg., come pt. pl., breken pt. pl., schul(le) plur., aus späterer zeit ist hinzuzufügen bare (pt. sg.), shalle (pl.) R 24. Die Suffolker testamente zeigen cam pt. sg. 1467: 41, pt. pl. 38, zu sculan den plur. shul 1467: 25 (sonst stets schall); in den Norfolker gilden erscheint bore pt.pl., ferner schullen, schal pl. (Schultz $40 \mathrm{f}$.), in späteren urkunden derselben grafschaft die sing. cam 1451 I 190, shul ebd. 189, die plur. shul ebd., shall(e) 1459 I 461, 463, 1482, III 282, 285 , in Worcester shullen $378,379,380$ u. oft, häufiger als shallen, shal 401, 404 u. ö.

(§ 259 a) In den Paston Letters findet sich zu beran das pt. sg. bare Wo I 404, 3JP II (383), III 198, das pt. pl. bare MP II (251), forbore III 136 . Von brecan ist belegt ein pt. sg. brak ClP I (540), brake Wo I 433, AP I (217), MP II 196, 238, 2.JP III (118), breke Norf. II (351), * I (134), in letzterem falle ist auch plural möglich. Neben dem pz. broken, brook 3JP II (349) u. ö. erscheint auch das (nach der ursprünglichen ablautklasse gebildete?) pz. breke 3JP II (357). Häufig sind beispiele für cuman. F kennt nur den sg. came I 314, 373, Wo sg. pl. came I 370 , II 175, 2WiP sg. come I $87(302,303)$, pl. cam I (505), MP sg. pl. cam (e), kam I 111, II 84, 85, III 431, sg. pl. $\operatorname{com}(e)$ u. ä. II $179,215,216,217,2 J P$ fast nur sg. pl. come III $115,116,128,160$ - cam pl. II (369) - , 3JP fast nur 
sg. cam III 140, 141 u. oft, pl. cam(e) ПI (122), III (35), 143 u. ö., selten pl. $\operatorname{com}(e)$ II $(3,122)$. $\mathrm{Zu}$ teran erscheint das pz. toryn 3JP III 144. Von sculan heisst der plural meist schall, shal MP I 179, EP III 278, 3JP III 48 u. oft, challe MP II 240 u. ö., seltener schull 2WiP I (297), xul MP I 110, choulle cholle II 240, 241, sholl 3JP II (347). Der sing. lautet auch schul 2WiP I 87, shul(l) MP II (194 u. ö.), MgP III 295, chull AP I (219), xul AP I 217, schol(l) MP I 531, II 83, Norf. II (16), shol 3JP II (347).

(§259 b) Bei Caxton ist der sing. stets regelmässig gebildet, nur für ae. cōm erscheint fast immer die analogische $a$-form, die auch für den plural herrschend geworden ist; (ebs. pt. sg. benamme 61). Die 3. ablautstufe ist nur erhalten in gelegentlichem shul RF 28, shulle 70; die übrigen beispiele von $\mathrm{RF}$ zeigen nördlichen ausgleich (bare 9, stale 18, tare 92, shal 4, 19 u. oft). Vgl. Römstedt 43.

\section{$\breve{e}$-reihe. Verba mit einfachem geräuschlaut} (klasse V).

(§ 260) Bei Wycliffe sind zahlreiche unregelmässigkeiten $\mathrm{zu}$ beobachten. Ganz regelmässig ist nur cweðan : pz. biquethun Ezech. XLVI 17 a. Von (for) biddan lautet das pt. sg. bad(de) forbad 2 Makk. II 4, Amos IV 7a, 1 Makk. XII 27 b, mit längung des stammvokals baad 1 Makk. XII $27 \mathrm{a}$, Apg. XIII $16 \mathrm{~b}$, das pz. bedun 1 Makk. II $64 \mathrm{a}$, Luk. XIV $8 \mathrm{a}$ usw., forbeden Hagg. I 10, Apg. XVI 6 b, Mark. IX 37 a, Luk. IX 49 b, $\mathrm{XI} 52 \mathrm{~b}$. Häufiger, namentlich in der jüngeren fassung, sind die entsprechenden formen von bèodan eingetreten (vgl. § 252). $\mathrm{Zu}$ ae. giefan heisst das pt. sg. zaf Matth. XVIII 32, zaue Matth. XIV 19, XV 36 u. ö., der plural lautet regelmässig zeuen nur Matth. XXV 37 a, meist mit dem vokal des singulars zauen Matth. XIII 8, XIV 19, 2 Kor. VIII 5, Off. IV 9 u. ö. und dem (unregelmässigen) pz. angeglichen zouen Matth. XXVI 67 a. Das partizipium erscheint nur selten regelmässig als zyuen Gal. III $22 \mathrm{~b}$, IV $15 \mathrm{~b}$, forzyuen u. ä. 2 Kor. II $10 \mathrm{~b}$, Jak. V 15 a, zeuen Ezech. XXXII 23 a, forzeuen Matth. IX $2 \mathrm{a}, 5 \mathrm{a}$, meist mit dem vokal der vierten klasse zouen $u$. ä. Matth. VII 7, 1 Joh. V 16, Offenb. VI 4 u. ö., forzouen Matth. XII 31, 32, Luk. XII 10. - Zu ae. gietan lautet das pt. sg. gat Hebr. XI 4, mit dehnung gaat Hebr. VI 15 a, das pt. pl. 
mit dem singularvokal gaten Hebr. XI 33, forzaten Matth. XVI 5, Mark. VIII 14, an das (unregelmässige) pz. angeglichen goten Dan. VII $22 \mathrm{~b}$. Das partizip ist meist regelmässig: geten ni. ä. Matth. XXV 20, 2 Kor. IV 1, 1 Tim. I 13 u. ö., bigetun Joh. I 14, Röm. VIII 29, Kol. I 18, forzete Ezech. XXII 12, forzeeten Baruch IV $8 \mathrm{a}$, selten, und nur in der älteren fassung, That es den vokal der vierten klasse: bygoten Ezech. XLIV 30 a, Mich. VI 7 a, Sach. XII 10 a. - Sehr mannigfaltig sind die rentsprechungen von ae. sêon. Das pt. sg. seah, sah ergab say Matth. III 16, IV 16, 18, 21, VIII 14, XII 22 (u. ö. in b), saiz Luk. II 26 a, Mark. VI 34 b (und oft in b), sayz Gal. I 19 a. Ebenso häufig sind jedoch entsprechungen von ae. *sēah (Bülbring s. $67 \mathrm{ff}$.), vgl. seez (über $20 \mathrm{mal}$ in $\mathrm{K}^{1}, 3 \mathrm{mal}$ in $\mathrm{A}$, nie in $\left.\mathrm{K}^{2}, \mathrm{M}, \mathrm{b}\right)$ Hosea IX 10, Matth. XXII 11, Ezech. VIII 2, syz Mark. VI 34 a, Luk. V 2 a, Joh. I 48a, siz Mark. V 38 a, VI $48 \mathrm{a}$ und meist so in $K^{2}$, ferner Dan. II $1 \mathrm{~b}$, Ezech. VIII $2 \mathrm{~b}$, 1 Makk. II $24 \mathrm{~b}$ und meist so in b. Durch angleichung an den plural sawen erklärt sich saw, sawz, sawe (in $\mathrm{K}^{1}$ die häufigste form, $3 \mathrm{mal}$ in $\mathrm{A}$, nie in $\mathrm{K}^{2}, \mathrm{M}, 5 \mathrm{mal}$ in b), z. b. in a Dan. II 1, 1 Makk. II 24, III 29, Matth. XIV 14, Ezech. II 9, in b Matth. IX 2, Luk. II 26, Joh. I 50, Gal. I 19, II 14. Dem plural liegen die ae. formen wests. sāwon, angl. sēgon und (selten) sāegon, sāgon zu grunde. Die $\bar{a}$-formen ergaben sawen, das in $\mathrm{K}^{\prime}$ weitaus die üblichste pluralform ist, z. b. Baruch III 20, Dan. X 7, Matth. XVII 8, sonst nur Ezech. XX 28 a erscheint. Aus sëgon, sø̄gon entstand sizen, syzen Matth. XXV 37, Mark. VI 33, Luk. VIII 34, IX 32, XVIII 15, 1 Joh. I 1, IV 14, Offenb. XI 11 (u. ö. in M), seezen 1 Makk. XVI 6, sayen u. ä. Matth. XX 34, XXV 38 (5 mal in $\mathrm{K}^{1}$ ), ferner Luk. XIX $7 a$. In b erscheinen folgende formen: sizen 1 Makk. III 17, Matth. II 9, 10 (13 mal), sien 1 Makk. VIII 18, X 64, Matth. IX 11 (9 mal), saien u. ä. Hab. III 10, Sach. X 2, 1 Makk. XIII 3 (16 mal). Im partizipium hat sich nur die anglische form gesegen erhalten, sie erscheint als seyn Matth. II 2, Mark. XI 11, 13, 1 Petr. I 8, Off. I 17, 19 u. ö. Daneben wird das adjektiv gesene als pz. verwendet, und zwar stets in A, meist in $\mathrm{K}^{1}$ (doch seyn Matth. II 2), selten in $\mathrm{K}^{2}$, wo belege jedoch äusserst spärlich sind, nie in $M$ und b, bis auf Matth. VI 18. Beispiele: Ezech. VIII 15, Baruch III 22, Matth. VI 1, 5, Gal. II 6. - Zu sittan erscheint ein pt. sg. sat Matth. 
XIII 2, XV 29, Offenb. IV 3 u. ö., mit dehnung (nur in a) sate Ezech. VIII 1, saat Joh. XIX 13, Hebr. VIII 1, Apg. II 3 u. ö.; pt. pl. se(e)ten Matth. XIV 9 a, Apg. VI $15 \mathrm{~b}$, saten Ezech. VIII 1 u. ö., Matth. IX 10, Mark. II 15 u. ö. - sprecan hat im partizip nur den ablautvokal der vierten klasse spoke, spokun Luk. XXIV 25, Joh. VI 64. Die übrigen formen lauten pt. sg. spac u. ä. Matth. IX 33, XII 22, 1 Kor. XIII 11, spaali Apg. IX 29 a, spake Offenb. XVII 1 a, pt. pl. speken Jak. V 10 b, Offenb. X 4 b, spaken Jak. V 10a, Offenb. X 3, 4 a u. o. - tredan erscheint Luk. XII 1 mit dem pt. pl. treden in der jüngeren, troden (mit dem vokal des nach der vierten klasse gebildeten partizips) in der älteren fassung, ferner pz. troden Ezech. VI 6 a, 1 Makk. III 51, wefan mit dem (ebenso entstandenen) pz. wouun Ezech. XXVII 7. Zu magan lautet der plural mown Joh. VII 34, Luk. XI 46. Schwache formen sind für ae. metan und wegan zu belegen, vgl. metid(e) Ezech. XL 5, 9,13 u. ö. in der älteren fassung (sonst matte ebd. XL 35, 47, 48 u. ö., meet XLI 1, in b stets mat), weyziden (b: weieden) Sach. XI 12.

(§ 261) Pecock bietet folgende belege: Zu biddan pt. sg. bade 138, 295, 446 u. ö., pt. pl. baden 446, pz. bede 7, 115, 116 u. ö., zu giefan das pt. sg. zaf 359 , zaue 211, 226, 289, pt. pl. zauen 281, 290, 305 u. ö., pz. zeuen u. ä. 67, 338, 368 u. ö., zouen 15, 16, 18 u. oft, zu gietan pt. gate 226 , pz. gete $(n) 67$, 68, 105 u. ö., bigete $(n)$ 246, 413, 497 u. ö., forzete 83 , zu sittan das pt. sg. saat 196, sate 300,359 , zu sẽon das pt. sg. size 34 , 425 , sauze 305 , pt. pl. sien 187 , sawen 246 , pz. seen 14,75 , 246 u. oft, zu sprecan das pt. sg. spak 202, spake 14, 488 u. ö., pt. pl. spaken 141, 246, 247 u. ö., pz. spoken 8, 12, 14 u. ö., zu magan den pl. may 184, mowe(n) 74, 184 u. oft.

(§ 262) Bei Chaucer ist wefan in die vierte klasse übergetreten, zu cweðan findet sich das pt. sg. quoth, neben sat steht mit dehnung seet; der sing. prät. dient bisweilen als plural; zu séon erscheinen die präteritalformen seih, sy, saugh u. ä. und die partizipia seyen, seene (t. Br. § $145 \mathrm{ff}$., 193, 198). Für Gower belegt Fahrenberg s. 398 das pt. sg. sigh. Hoccleve bildet die pt. sg. byqueeth, speek und spak, zu forgietan und giefan partizipia nach der vierten klasse (Vollmer 220), zu sẽon erscheinen die reime sy pt. sg. : I MP 110/6, ebs. 106/310 u. oft, say : way 233/477, ebs. $138 / 780$ u. ö., sy (2. sing. pt.) : I 
RP 394, sye (pt. pl.) : awrye MP 98/77, seyne (pz.) : agayne RPr 3032, seen (pz.) : bene RP 2528; im plural von magan ist may herrschend geworden. Aus Lydgate belege ich sate (pt. sg. : gate sbst. AG 1547), bade (pt. sg. : sade 1560), awroke (pz. nach klasse IV, : broke pz. - nicht ganz sicher - AA I 729), may (pl. : day Marg. 193 u. ö.) und zu sẽon pt. sg. say (: assai TGl 694), sawe (: strawe AG 559), 2 pers. sy (: by AG 1740), pz. sayne (: certayne AA II 1542 u. oft), sene (: kene AA III 1570 u. ö.). Bokenam hat im pt. sg. say und sy (Hoofe $249 \mathrm{f}$.), im pt. pl. sey (: aweye VI 604, : preye III 346$)$, im pz. seye (: deye V 132, : eye < eage IX 235), seyn (: ageyn V 245 u. ö., : certeyn XI 285) und sene (: grene X 840), zu magan noch pl. moun : resoun III $788 \mathrm{u}$. oft. Aus den werken der übrigen Chaucerschüler ist bemerkenswert gave pt. pl. : save : haue Gen. 3313, get pt. sg. : grete Part. 2541, begett pt. sg. : mett pz. Wade 504, get pz. : grett Part. 1690, 4319, sey pt. sg. : way CL 693 , sie pt. sg. : certainely FlL 78, : skie 194, save pt. sg. : felawe CL 1030, : lawe : awe Gen. 1612, : draw Part. 609 u. ö., Wade 154 u. ö., saw pt. pl. : gnaw < gnagan K. Orl. 229; pz. seen und sayn sind häufig.

(§ $262 \mathrm{a})$ Die volkstümlichen dichter bieten für sēon die bereits bei den Chaucerschülern belegten reime, für das pt. pl. sey : dey < daeg Stat. Jer. 567, se (lautwert i) : mandć ebd. 49, sye : wittirlye Lon. XVIII 315, ebs. XXV 185, XVI 139, size : victorie : die : companye Lamb. II 54/421; von anderen verbalformen führe ich an pz. begete : strete Beryn 2943, forzete : behete (Ms. behizte) 1125, ebs. 3993, forgote : dote Lon. XXI 297; seet (Ms. sate; pt. sg. zu sittan) : fete Stat. Jer. 813, : thrett < preatian Beryn 591, sate pt. pl. : gate < geat (unsicher) Ryman CXI 4; zeuede (gab) : leuede (lebte) Eth. 347.

(§ 263) Bei Capgrave sind zu sẽon folgende formen belegt: pt. sg. say : way Kath. III 360, 897, syze : eye (wohl $i$ ) II 1284, ebs. see : beute II 1312 ; 2. pers. sing. say : day : aray V 1439; pt. pl. seyn : seyn < secgað : ageyn I 670, : playn : slayn III 1383, pz. seyn : sayn < secgan : certayn III 453, ebs. 944, sene : qween II 381 u. ö., : sheen III 560 . Chr. schreibt für das pt. sg. say, sey 25, 29, 30, 47 u. ö., saw 248 (zweimal), 253, pt. pl. sey 58, 108, 226 u. ö., pz. seyn 58, 93, 151 u. ö., sey 115, 178. Ferner sind $\mathrm{zu}$ erwähnen : pt. sg. beqwath 235 , beqwathe 130, 156, pt. pl. beqwathe 71 ; pt. sg. gaf 106, 156, 179 u. ö., 
gave 106, 116, 129 u. ö., pt. pl. gave 183, 186, zove 21, pz. gove 9, 105, 177 u. ö., zove 104. - pt. sg. gat 90, 131, 261, begat $20,21,24$ u. oft, pt. pl. gote 201, begotin 15, pz. get 236, gote 155,224 , begoten u. ä. 220, 237, 263 u. ö., forgoten 177. pt. sg. sat 37, 263, 304 u. ö., pt. pl. sat 190, 253 (zweimal). pt. sg. spak 27, 32, 310 u. ö., pt. pl. spak 302, spoke 74, pz. spoke 6 u. ö., dazu pt. sg. spake (: I lake) Kath. III 1177, : (bak) IV 352, pz. spoke (: broke) III 1427 - pt. sg. (for)bad 220, 250, 260 u. ö., forbade 65, 291. - pz. wove 204.

(§ 264) In den Londoner urkunden ist von sẽon belegt pt. pl. seyen, pz. seen, forseyne; mit dem partizip nach der vierten klasse kommen vor bicweðan, giefan, gietan, sprecan; $\mathrm{zu}$ cweðan gehört pt. sg. quod, zu magan der pl. mowe und may (Morsbach $139 \mathrm{ff} .$, 149). In den späteren staatsurkunden erscheinen seeyn (pz.) R 13, forseen $80-$ pt. sg. gave $\mathrm{E} 71$, $\mathrm{R} 71$, yave E 60,77, pt. pl. yave R 61, pz. gyven R 12, geven $\mathrm{R} 18,22,23$ u. ö., yeven $\mathrm{E} 15,16, \mathrm{R} 62$ u. o., yoven $\mathrm{R} 16,75$, 77 u. ö., E 16, 17, yuven E 61, 64, 76 - pz. begoten R 44, 45 - pz. spoken R 50. - Plur. may R 50 u. ö. - In den Suffolker testamenten kommen vor pz.beqwethyn 1481: 60 - pt. sg. gaf 1467: 23, 31, pz. gevyn 1470: 50, yeuen 1481: 68, youyn 61, youen 1482: 55, gove 1467: 31 - pt. sg. get 1467: 19, pz. forgete 1481: 55 , goten 1481: 66 , begotyn u. ä. 1467: 24, 1481: 56, 57, 1482: 52 - pz. spoke 1467: 27. - plur. may 1467 : 30 u. ö. - Für die Norfolker gilden führt Schultz (s. 40 f.) an sowen $(!)<$ sāwon, dazu pz. seyne $\mathrm{BL} 87$, mowe $\mathrm{u}$. ä., may in der grossen urkunde. Die späteren schriftstücke derselben gegend bieten noch pz. unbiquothen 1482 PL III 288; pt. sg. yaf II 224, yave 1451 I 191, pz. yeven 1482 III 283, yove(n) 1451 I 189, 1465 II 224, pz. goten 1482 III 286; may ebd. 282. Aus Worcester sind zu belegen seyn pz. 380, yeven pz. 406 u. ö., spoken 407, may (pl.) 392.

(§ 265) In den Paston Letters kommen vor: Von biddan pt. sg. bad 3JP III (357), bade MP II (192), Devon I (85); pt. pl. bade MP II 215, Devon I (85), bad AP I 217, 3JP III 31, MP II $(131,63)$ - giefan : pt. sg. gave MP III 23, 2JP III 101, 3JP III 40, gaff(e) 1JP I 348, 2JP III 187, zave MP I 109, yaffe MP II 215, auch geff 1 WiP I 25, yef * I 390; pt. pl. gave 2JP III 199, 222, 3JP III 43, 217, 218 u. ö., gaffe MP II (342), 2JP III (94), selten zeve MP II (132), yeve * III 
(97), geffe AP I (114), yovyn Norf. II (260); pz. gevyn MP III 194, 2JP III 128 (u. ö.), 3JP II (343 u. ö.), zevyn AP I 217, gef 1JP I 234, gifen Norf. I 117, yovyn MP II 215, zovyn MP I 112, 113, govyn 3JP III 220 (u. ö.) - gietan : pt. sg. gat 3JP III 73, gate Wo I 375, MP I 109, gete MP I (68), +3WiP III 344, gote * I (133); pt. pl. gett MP II 239, gate 2JP II (381), gaate ${ }^{*+}$ III 334 , forgate 2 JP III 166, got * I (135); pz. gyte MP II 179, getyn 1JP I 518, gete MP I (355), geth (e) 2WiP I $(303,494)$, begete $(n)$ F I 93, forgetyn 2JP III 187, 3JP II (305), forget MP I 178, - hierher gate Norf. II (205)? vgl. §47-; goten MP II 217, MgP III 292, gotyn 2JP III (83 u. ö.), 3JP III 68, 155, 218 u. ö., MgP III 295, begotyn 2JP III 188, forgotyn 2WiP II 109, 2JP II (321) und schwach foryeted F I (170) - seon : pt. sg. sey 1 WiP I 25, 2WiP I (303), MP II 83, I (438), II (250), seigh * I 136, $s y(e)$ MP III 124, 2JP III 104 u. ö., 3JP III 69 u. ö., syghe $2 J P$ III 177, saw 3JP III 43, Norf. I 513, II (146), * III (182); pt. pl. sey MP I 113, 3JP II (319), sye 3JP III (35), sawe Devon I (85); pz. seyn MP II 202 (u. ö.), 2JP II (340 u. ö.), 3JP II (401 u. ö.), EP III 279, sene $2 \mathrm{WiP}$ (?) II (5), seen Norf. II (69). - sittan : pt. sg. sete Linc. I (97); pt. pl. sat 3JP II (357), pz. auffälliger weise satyn Norf. II (50), satte II (205) - vgl. pz. gate. - sprecan : pt. sg. spak 2WiP I 515, MP I 179 u. ö., 3JP III 68 u.ö., spake MP I 109 u. ö., 2JP III 85 u. ö., Norf. II 55, speke 2JP III (118), Norf. I (315), II (333, 351), Linc. I (98), spok, spooke ClP II (42), MP I (43), II (63), 3JP II (358), III (54); pt. pl. nur spak 2WiP I 492, MP I 257 u. ö.. WaP III 246, spake MP III 431 u. ö., 3JP II (347) und spoke(n) MP II (130), * I 386; pz. noch speke(n), spekyn 2WiP I 87, MP I (77 u. ö.), 2JP III (153), 2JP II (357), meist jedoch spoke(n) MP I 257 u. oft, 2JP III 21 u. ö., 3JP II (401 u. o.). Die form spoken in my Lorde desired you to come and spoken with hym Norf. II 55 fasse ich ebenfalls als pz. und ergänze davor $a<$ have, zumal der schreiber öfters selbstverständliche wörter unterdrückt. - tredan : pt. sg. trad AP I 217. - magan : pl. meist may Wo I 37, MP II 82, 1JP II 209, 2JP III 222 u. ö., selten now(e) AP I (59), * III (97).

(§ 266) Bei Caxton erscheinen im pt. sing. einige anscheinend gelängte formen: gate $\mathrm{RF} 8,16,35$ u. ö., brake 28, 
116 neben brak 15, trade 105 neben trad 15; das pt. sg. zu sêon lautet stets save 7, 15, 16 u. ö. Im plural ist überall die singularform herrschend geworden, so in bad, gate, gaf $(e)$ u. ä.; ferner stacke (ae. stecan) 16, spake 60, spack 30, satte 27 (sattest 96); dazu sawe. Das pz. ist regelmässig nur in gyuen $\mathrm{RF} 12,19,22$ u. ö., geuen 9, forgeten 90, daneben erscheinen youen, goten, spoken; broken 21, 39, $56 \mathrm{u}$. ö. Das pz. zu sẽon heisst stets seen. Vgl. Römstedt $43 \mathrm{f}$.

\section{$\bar{e}$-reihe (klasse VI).}

(§267) Auch aus dieser ablautreihe sind einige übertritte zu anderen klassen erfolgt, namentlich zu den reduplizierten verben. Bei Wycliffe lautet das pt. sg. von slēan zwar in der jüngeren fassung regelmässig slow 1 Makk. II 24, III 11, 2 Makk. XII 6, XIII 15 u. ö.; die ältere fassung dagegen hat nur Dan. V 19, 1 Joh. III 12 slow(z), sonst slew(z), das doch wohl einem ae. *sleow entspricht. Dieselbe scheidung zeigt der plural slewen und slowen Sach. XI 5, 1 Makk. I 60, V 51 u. ö. Ebenso erscheint 1 Makk. V 51, XI 38, Offenb. XII 4 in a : drewz (vgl. die schreibung knewz < cneow 1 Makk. XIII 17 a, 2 Makk. XII 5 a u. ö.), in b: drow (sing. u. plur.). Uebertritt in die $\breve{e}$-reihe zeigen die pt. pl. bitaken Apg. XV $30 \mathrm{a}$, forsaken 2 Petr. II $15 \mathrm{~b}$ und das pz. toke Dan. X 1 a (nach klasse IV wie woxe). - ae. weaxan hat nur wenige regelmässige formen: pt. pl. woxen 2 Makk. XI 9, Matth. XIII $7 \mathrm{~b}$, Luk. XXIII 5, 23, pz. waxen Dan. VIII 8a, wexen Matth. XIII $32 \mathrm{a}$, wexun 2 Makk. VI $18 \mathrm{~b}$, wexe Ezech. XLVII $5 \mathrm{~b}$ (vgl. Sievers § 392, anm. 7). Oefter zeigt es den ablaut der reduplizierten verba: pt. sg. wex(e) Jon. I 13, 1 Makk. IX $66 \mathrm{~b}$, Luk. Il $40 \mathrm{~b}$, pt. pl. wexen Sach. I 8, 10, 11, Matth. XIII 7 a, Joel I 17 b. Häufig sind formen nach der IV. klasse: pt. sg. wax(e) Ezech. XXVII $32 \mathrm{a}$, Luk. XIII 19, Dan. X 19 a, Luk. Il 40 a, Apg. VII 17 a, pz. woxen Dan. IV 19a, 2 Makk. VI $18 \mathrm{a}$, Matth. XIII $32 \mathrm{~b}$. Am gebräuchlichsten sind schwache formen: wexide Jon. IV 10, Apg. XIX 20, Dan. IV 19a, X 19 b, Joel III $13 \mathrm{~b}$, wexidist Dan. IV $19 \mathrm{~b}$, wexiden Dan. IV 30 , Obadja 7, Mal. III 13, 1 Makk. VI 6, Ezech. XIX 10a, XLVII $5 \mathrm{a}$, Joel I $17 \mathrm{a}$, einmal waxede Apg. VII $17 \mathrm{~b}$. - Schwache formen zeigt auch ae. grafan : pt. sg. grauede Hab. Il 18, 7. raued Ezech. XL 34 a, XLI $20 \mathrm{a}$, die jüngere fassung 
schreibt a. a. 0. grauun, ähnlich auch in a grauen, grauein Dan. XI 8, Micha V 12, 1 Makk. XIII 29 u. ö.

(§ 268) Bei Pecock wird grafan bis auf pz. graue 169 stets schwach gebraucht, vgl. pz. graued 138, 139, 169 u. ö.; $\mathrm{zu}$ weaxen begegnen pt. sg. wexe 342 , wexid $322,334,339 \mathrm{u}$. ö., pt. pl. uaxiden 243. Das pz. zu sléan lautet slayn 157, slein 246.

(§ 269) Chaucer hat zu weaxan formen nach der IV. und VII. klasse, zu slēan die pz. slawen und slayn, zu hebban das pt. sg. haf nach der $\breve{e}$-reihe (t. Br. § 149); letzteres erscheint auch bei Hoccleve (: staf stab) MP 172/912. Lydgate bietet slawe (: drawe 2 M 735, ebs. Th 358 a, MP 253/259) und slain (: desdain Th 374 c, ebs. AG 1568, AA III 15 u. ö.); das pt. sg. heisst slow (: how Th $362 \mathrm{~b}$ ); ähnliche doppelformen für das pz. erscheinen bei den übrigen Chaucerschülern (und den volkstümlichen dichtern). Ferner sind bemerkenswert sly nach klasse VII (pt. sg. und pl.) in Part. (Hattendorf 39), ebs. drewe : pursue Wade 59, 1660, : trewe 1989, : trew : uertew Part. 3047 und forsolie (pz.!) : he toke Wade 2243. Aus der volkstümlichen litteratur sind anzuführen sloon (pz.) : ychoon Unz. T. 246 (vgl. §59), die präterita heve, hevyn zu hebban in Ed. Eth. (Fischer 214, vgl. sly zu slöh) und stond zu standan (nach klasse III) : fond (pt. pl.) Stat. Jer. 532.

(§ 270) Bei Capgrave lautet das prät. zu dragan meist regelmässig drow 191, 195, 201 u. ö., seltener drew 47, 144, 281. $\mathrm{Zu}$ slean heisst das pz. nur slayn 188, 189, 190 u. ö., zu weaxan das pt. sg. wex 141, 176. fléan bildet ein pt. sg. flay (vgl. sly, heve) 33, pz. flayn 61 .

In den Londoner urkunden kommen vor pt. pl. drogh und drewe, pz. slayn, sworen (Morsbach $139 \mathrm{ff}$.); gegen ende des jahrhunderts ist das schwache pz. understanded $\mathrm{R} 12 \mathrm{zu}$ belegen, ferner das pz. slayne $\mathrm{R} 68$, das auch in einer Norfolker urkunde von 1451 (PL I 190 slayn, pt. slow ebd.) erscheint. Zu grafan belege ich pz. gravyn Suff. 1467: 15.

(§ 271) Die Paston Letters bieten zu dragan das pt. sg. withdrew 3JP III 341, pt. pl. drew 256, zu sléan das pt. pl. slowe Suff. I (429), das pz. slayn(e) MP I 110, 2JP III (162), Suff. * I $(429),{ }^{*}$ II $(13,118)$; zu swerian erscheint auch nach klasse IV ein pt. sg. sware * I (345) - pz. stets swore u. ä. -, zu takia nach klasse IV ein pt. sg. take 2JP II (41) und ein schwaches pz. taked Wo I 432, zu weaxan pz. waxse MgP III 215. 
Bei Caxton sind zu den reduplizierenden verben übergetreten weaxan, wascan, meist sléan, auch dragan und hebban; zu swerian und weaxan werden formen nach der 4 . klasse gebildet (Römstedt 44); nur einmal ist belegt das pt. pl. swore 37 .

\section{Reduplizierende verba (klasse VII).}

(§ 272) Bei Wycliffe sind bemerkenswert ae. feallan, fealdan, hätan. Ersteres wird gewöhnlich regelmässig abgelautet: pt. sg. fel, fell(e) Ezech. VIII 1, Dan. II 46, Apg. XX 9 u. oft, pt. pl. fellen Ezech. XXVII 34, Hosea VII 7, Offenb. IV 10 u. oft, pz. fallen Hosea XIV 2 a, Offenb. XVII 10 a, bifallen 2 Makk. V 18a. Daneben werden schwache, meist aus dem prät. gebildete formen verwendet: pt. sg. felde Joh. XI 32, Apg. V 5, Luk. XVII 16 a, Apg. XV 16 a, Offenb. XIV 8 a, Apg. X $10 \mathrm{~b}, 44 \mathrm{~b}$ und oft in b, bifelde 2 Petr. II $22 \mathrm{~b}$, pt. pl. felden 2 Tim. II 18, Hebr. XI 30, Luk. XVI 21 a, Röm. XI 22 u. ö., felliden 1 Kor. X $11 \mathrm{~b}$, falliden 1 Tim. VI $21 \mathrm{a}$, pz. (bi)feld 2 Makk. V 18 b, Offenb. XVII 10 b. - fealdan ist nur im partizip, und auch da spärlich vertreten. Regelmässig sind foldyn, foldun Ezech. XLI 24, II 9 a, schwach fo(o)ldid 2 Makk. X 26 a, Ezech. II $9 \mathrm{~b}$. - Zu ae. hātan belege ich prs. bihotinge 2 Petr. II 19 a, ferner neugebildet nach dem pt. byhetith Hebr. XII 26, byheten 2 Petr. II $19 \mathrm{~b}$, bihetynge Dan. III 36, 1 Tim. II 10, pt. sg. bihizt(e) Matth. XIV 7, Röm. I 1 a, pt. pl. bihizten Mark. XIV 11, pz. bihote Röm. I $1 \mathrm{~b}$. - Zu sāwan erscheint ein pz. sewen Matth. XXV 24a. - 'Sie hängten' heisst hangide(n) 1 Makk. I 64, IV 51, 2 Makk. XV 35.

Bei Pecock hat das pt. zu feallan stets $i$ im stamm: pt. sg. fill, fille, bifille 286, 447, 448, pt. pl. fillen 199, 204, 248 u. ö. hōn hat im pt. stets $e$ : heng(e) 139, 199, 261, hengen 22 (nur intransitiv).

(§ 273) Chaucer bietet an bemerkenswerten formen fel und fil zu feallan, heet und highte $\mathrm{zu}$ hätan, heeng $\mathrm{zu} h \bar{o} n$; slēpan und wepan schwanken zwischen starker und schwacher flexion (t. Br. $\$ 130,134 \mathrm{f}$.). - Aus den reimen seiner schüler sind zu erwähnen fill : hill Lydgate Th $361 \mathrm{a}$, ebs. Hoccleve RPr 3705, Gen. 1219 u. ö, heht : ryht : syht Bokenam V 95, wepe pt. sg. : kepe Lydg. Th $361 \mathrm{a}$, pt. sg. hyng : thing : ryng Gen. 5236, prs. (< an.) hynge : pykynge Bab. B. 148, pt. sg. 
honge (nach der 3. ablautreihe, intr.) : songe < sungen Wade 2145. In der volkstümlichen litteratur erscheint fille (: vntille) Lon. XXVI 103 u. ö.; beheete (Ms. -hizte) pt. : forzete pz. Beryn 1126; für hōn ist oft das an. hengja eingetreten, z. b. heng : swing $<$ swingan : betyng : king Lamb. II 28/201, : levyng Audelay 1, : drynke ebd. 61. (Diese formen sind häufig im norden, vgl. hyng : wederlyng : spryng Susanna 101, ebs. Henrison 199/167, ähnlich 115/178, 210/19).

(§ 274) Capgrave hat für das präteritum von $h \bar{o} n$ im reim hyng (intr. : yinge Kath. IV 1923), für das partizip hong (trans. : long : wrong IV 1852); in Chr. pt. (intr.) hing 306, ferner nach der 3. klasse mit beeinflussung durch das pz. (trans.) hung 55, 146, 214, pt. pl. (trans.) hung 169, pz. (ebenfalls trans.) hang 188, hange 162, hangen 190, 287, $316 \mathrm{u} . \ddot{0}$., daneben hanged 154, 286. Zu hātan lautet das pt. (be)hite 17, 31, 297 u. oft, behote (nach dem pz.?) 290, das pz. regelrecht behote 208, 307, daneben (be)hite 264, 307, auch als prs. wird hite gebraucht 23, 37. feallan hat als pt. stets fel(le) 36, 37, 58 u. oft. $\mathrm{Zu}$ slōepan finden sich bis auf ein st. pt. slepe 34 nur schwache formen (slept 82, 85), ebenso zu sāwan (pt. sowyd 131).

Die urkunden bieten an bemerkenswerten formen nur felle pt. pl. R 22, helde(n) pz. (Schultz 40), (be)hoten (pt. pl.) Norfolk N 27 (also prs. *hīten), (pz.) 14, 30, 37 u. ö.

(§ 275) Aus den Paston Letters ist zu erwähnen ae. feallan mit dem pt. sg. fell(e) 1JP I 252, Norf. III 73, 168 u. ö., fille $2 \mathrm{WiP}$ III 112, MP II 99 und dem pt. pl. fyll(e) 3JP III 43,50 , Norf. II (205); ferner zu wealcan, das um diese zeit sonst schwach flektiert wird, das pt. pl. welk MP I 111; zu hleapan pt. lyepe 2JP III (235).

Bei Caxton erscheinen zu slēpan noch einige starke formen (Römstedt 44), hleapan flektiert noch stark, vgl. leep 10, 28, 44 u. ö. Das pt. von feallan heisst meist fyl 22, 23, 27 u. ö., selten bifelle 91, byfel 97. Das pt. von ae. hōn lautet henge (intr.) $\mathrm{RF} 55$, 96, 108, henge (trans.) 51, seltener hynge (intrans.) 100, hynge (trans.) 39, 47, daneben hanged $67,101$.

(§ 276) Betrachten wir noch einmal die ablautverhältnisse der starken verba im zusammenhange, so zeigt sich, dass die ae. reihen ihre festigkeit völlig eingebüsst haben. Häufig verschieben sie sich unter einander, so nach der 3., besonders aber 
nach der 4. klasse hin (pt. $a$, pz. o); der partizipialvokal $o$, den ausser der 4. noch die 2. und ein teil der 3. reihe besass, dringt auch in die übrigen klassen ein (yoven, woxen; bereits ae. brocen, sworen), wird somit eine art normalvokal des partizipiums wie (in geringerem grade) $a$ normalvokal des präteritums wird. Daneben machen die reduplizierenden verben beträchtliche eroberungen (sty, wex, drew usw.), und auch zur schwachen flexion treten starke verba in grosser zahl über, meist ohne, aber vereinzelt auch mit ablaut des präsensvokals (writiden, risid, zeldide, keruyden, yoldyd, metid, foryeted, taked), so dass der historische unterschied zwischen beiden konjugationsarten fast verschwindet; denn auch in der schwachen flexion ist vokalwechsel nichts seltenes (reden-radde, leden-ladde, levenlafte, reven-rafte usw.).

Noch mehr sind die alten ablautreihen durch ausgleich der einzelnen stufen zerstört worden. In nördlicher weise wird das pt. pl. durch den sing. ersetzt, in westlicher art dringt der partizipialvokal in das präteritum. Am meisten hat sich der nördliche ausgleich geltend gemacht in der 4. und 5. klasse, wo das pt. sg. durch fakultative dehnung des stammvokals denselben vokal wie das pt. pl. entwickeln konnte; verhältnismässig am wenigsten ist er in der 1 . klasse vorgedrungen.

(§ 277) Ein vergleich der einzelnen denkmäler unter einander wird dadurch erschwert, dass die hierher gehörigen formen in manchen quellen nur spärlich, in den urkunden so gut wie gar nicht belegt sind. Doch zeigt sich deutlich, wie der verfall der ablautreihen während des 15. jahrhunderts zunimmt; nördlicher ausgleich ist in der jüngeren Bibelfassung häufiger als in der älteren, und bei Pecock häufiger als in jener, ebenso bei Lydgate und Capgrave öfter zu finden als bei Chaucer, wozu auch der nördlichere heimatsdialekt der beiden letzteren mit beigetragen haben mag. Ferner ist die Oxforder gelehrtensprache in diesem punkte fortschrittlicher als die östliche gruppe: Wycliffe hat häufiger ausgleich als Chaucer, Pecock (wo genügend belege vorhanden sind, wie in der 1. klasse) häufiger als Capgrave; sodann scheint die Londoner urkundensprache weiter vorgeschritten zu sein, als Chaucer, wenn wir aus den beispielen der 5. ablautreihe (cwoden, goven, goten gegenüber yiven, geten) auf die in den urkunden kaum belegten übrigen schliessen dürfen. Auffällig ist die 
relative häufigkeit westlicher formen in Norfolk (sg. funde, brok, pl. bore).

Caxton stimmt auffällig mit den Oxfordern überein, sowohl was die häufigkeit des ausgleichs anbetrifft wie in einzelheiten, so hat er wie jene fast stets $\operatorname{cam}(e)$, selten $\operatorname{com}(e)$.

\section{Bildungsweise der schwachen verba.}

(§ 278) Das suffix-i der alten $\bar{o}$-klasse ist in unseren denkmälern in einigen resten erhalten, die an südliche quellen des 14. jahrhunderts erinnern: schonye < scunian 2 Tim. II 16 a, Tit. III 10 a, avorthi < geforpian Pecock 306, 336, 377, 562 (hierher auch quykee, lothee usw.? vgl. § 165), asky < äscian (: sekyrly) vKath. 422, wari < wyrian, wiergan (: myscary : tare $=$ ne. tarry) PlSa 557, loki W 102/28. Auf frz. -ir beruht seruy (: redy) Ed. 1223, auf einer vermischung afz. und ae. formen Chaucers astonien und harien (t. Br. § 174); letzteres findet sich ausserdem noch bei Hoccleve (Vollmer 218), ersteres als stuni noch heute in Oxfordshire (Ellis 126); in Somerset dazu mit analogischem $-i$ ne. laugh, talk, prate, wash (Ellis 148, 150, 153).

(§ 279) Von verben der $\bar{e}$-klasse kommen noch vor ae. habban, libban, secgan. habban erscheint allgemein als have (ae. *hafian), danach auch 3. sing. havyth MP I 257, have (vgl. $\S 186)$ MP II (385), Norf. II 24, 60, 161. Ebenso ist libban bis auf wenige reste (§169) in *lifian untergegangen, secgan an die 2. 3. sing. (sagst, sagd) angebildet worden, bis auf sig PISa 358.

Ueber licgan, bycgan, lecgan vgl. § 204, über verba romanischen ursprungs auf -issen $\S 189$.

(§ 280) Oft ist das lautgesetzliche präteritum durch ein neues, dem präsens angebildetes, ersetzt worden. Hierher gehören dredde, redde usw. (vgl. § 34), ferner makid bei Chaucer (t. Br. § 173), Beryn (: ransakid 3651), Lamb. (: nakid : rakid II 89/21), hauened (nach inf.) L 45; so auch mit bindevokal infeelid, feleden Pecock 448, meened 345, 448, 494. Me. cacchen $<$ frz. cachier hat nach me. lacchen < loeccan die pt. cacchid und caute entwickelt, so Chr. 189.

Doppelte präteritalbildung liegt vor in ouztiden (sollten) Pecock 157, hadyd (hatte) 2JP III (195), taughted (lehrte) RF 103.

Anglia. N. F. XII. 
(§ 281) Starke formen haben neben den schwachen entwickelt ae. mearcian und werian; für ersteres vgl. pz. morkyn 2JP II (293, 294), 3JP II (122), Norf. Urk. 1482 III 285, für letzteres pz. worn(e) Pecock 347, 543 (werid 532), pt. pl. weren Chaucer (t. Br. § 144), pt. pl. ware : bare adj. : fare FlL 340, pt. sg. were Capgrave Chr. 103 (wered 70, 75, 313), pt. pl. ware 3JP III 141, pz. woryn 2JP III (95), 3JP III 73, pt. sg. were, veryd Suffolk 1467: 15, ware, worn Caxton (Römstedt 43).

\section{Flexionsendungen.}

Ueber apokope des $-e$ ist bereits $\S 155 \mathrm{ff}$. gehandelt worden.

1. person singularis ind.

(§ 282) $\mathrm{Zu}$ erwähnen ist nur, dass in den Paston Letters die 1. pers. sing. gelegentlich in nördlicher weise an die (2. und) 3. sing. angeglichen wird, vgl. ... like as I has wretyn to hym F I 94, ebs. I never attemptid to procede ageyns hym ... and desired ... and hath be redy 1JP I 233. Noch Wyatt hat einmal has (Hoelper 53), und formen wie I knows, writes, means sind noch heute in mehreren grafschaften des südlicheren Englands zu belegen (Ellis s. 44, 100, 178 u. ö.), werden jedoch kaum auf nördliche einwirkung zurückzuführen sein.

\section{2. person singularis ind.}

(§ 283) Die 2. person singularis geht im allgemeinen auf -st aus. Die nördliche endung $-s$ findet sich ausnahmsweise bei Chaucer (Haus der Fama III 818), auch in der Chaucerschule, so weit ich sehe, nur einmal: haste : grace : was : place Dor. 271. In der volkstümlichen litteratur ist $-s$ dagegen nicht ganz selten. Vgl. Lon. has : plase XXIII 42, Lamb. I has : was : alas : trespase 149/47, flees : vanytees : leese inf. : chese inf. 187/281, has : grace : face : taas $={ }^{*}$ takest $197 / 458$, failis : batailis 202/610, tendis : ameendis pl. : pou wendis : freendis II 114/2, Audelay has : trespace 1, gase gehst : face 68 . Capgrave hat gewöhnlich die endung -st: hast 50, 80, 141, nedist 50, knowist 210 usw., jedoch einmal $-s$ : tredis 158. $-s$ ist übrigens noch im 16. jahrhundert zu finden (Hoelper 54).

(§ 284) Die 2. pers. sing. der präterito-präsentia hat gewöhnlich die endung $-(s) t$; jedoch erscheinen auch öfters endungslose formen in nördlicher art. Dreimal findet sich thou shal in der älteren fassung der Bibel (Ezech. VIII 6, 15, 
Mark. XII 30); von den werken der Chaucerschule haben endungslose formen CL (thou can : beganne 462), Part. (Hattendorf 43) und stets Bokenam (thou shal : al : fynal I 550 u. ö., kan : Flauyan I 993, may : day VII 131 u. ö.). Häufig sind sie in der volkstümlichen litteratur, vgl. schalle : withal Lon. XV 493, ebs. vMarg. 181, Lamb. II 1/15, can : on Beryn 2146, may : pray Audelay 52 usw. Ihnen reiht sich Capgrave an, der nur einmal thou schalt 313 bietet, sonst stets endungslose formen: schal $(l) \mathrm{Chr}$. 31, 56, 141 u. ö., may 314, ebs. in Kath. shal : reall III 182, : wall III 299, can : man IV 1157. - Caxton hat einmal shal, sonst stets shalt, mayste usw. (Römstedt 48f.).

\section{3. person singularis.}

(§285) In der Oxforder gelehrtensprache geht die 3. person ausnahmslos auf th aus. Auch in der poesie ist -th weitaus das gewöhnlichste, doch verwendet schon Chaucer gelegentlich die nördliche endung - $s$ (t. Br. § 185, B. Duch. 257), die dann auch bei vielen seiner schüler erscheint. Zwar folgt Hoccleve getreu dem südlichen sprachgebrauch, jedoch schon Lydgate reimt specifies : fantasies $\mathrm{Th} 363 \mathrm{~b}$, manaces : places AG 61, leres : baneres Th $363 \mathrm{c}$, shewys : thewys ScPh 29, techys : spechys : lechys $\mathrm{ScPh} 1222$, ebs. Burgh (feynes : peynes 1748), Rem. L (festes < foested : ghests < giestas $541 \mathrm{a}$ ), K. Orl. (hase : frz. case 15, ebs. 99 , dyes : arises inf. [=arīs] 39, lise : devise inf. 59 u. ö.) und Rich. Ros (Gröhler 17). Noch häufiger sind die nördlichen formen in der volkstümlichen litteratur. Vgl. Lonelich gos : (a)ros XVI 30, XXVI 414, : clos XXXI 366, sayes : dayes pl. XXIII 500, XXVII 309, wones : persones XVII 294, tellis : ellis XXX 664, Beryn ridis : sidis 1636, hies eilt : vlyes Fliegen 2350, hondis (= ne. handles) : sondys plur. 3946, Lamb. stondis : boondis pl. : hondis pl. : sondis pl. I 181/189, aylis : batailis pl. : asailis 3. sing. 202/612, has : plase : was usw. 213/142, lijs (zu licgan) : paradijs : rise inf. : seruice II 2/29, hatis : zatis pl. 53/372 usw. (sehr häufig), Audelay has : grace 69, avaylis : naylis pl. 57/56, bygynnes : synnes pl. 55, PlSa hase : space 463.

Capgrave hat nur einmal die endung $-s$ in welles (3. sing. zu weallan) : welles (pl. zu wella) I 788, sonst stets in Kath. und Chr. formen mit -th. 
Die Londoner urkunden zeigen ebenfalls nur einmal $-s$ (Morsbach 134), gegen ende des jahrhunders erscheinen nur has $\mathrm{R} 17$, beres 48 , thankes 48,49 . Häufig ist es in den Norfolker gilden, jedoch nicht in den urkunden von Norwich (Schultz $37 \mathrm{ff}$.). Im 15. jahrhundert ist es in Norfolk und Suffolk überhaupt nicht zu belegen.

Dagegen ist $-s$ nicht selten in den Paston Letters, es fehlt jedoch gänzlich bei 1WiP, 2WiP, MP und 1JP. F schreibt has I 94, sendis, standys I 93, haldis, sais I 94, EP thynkks (I 71), prays III 27, has III 278, semys, makys, servys, hasse † III 382, 383, 2JP shewys II 295, semys III 101, helpys III (121), 3JP sendys III 73, II (348), preyes III 73, waytys III (46), thynkys III 57 u. ö., MgP intendes III 293, Wo qwyts I (294), Norf. telles III $72 \mathrm{u}$. oft und einigen anderen schreibern ziemlich unsicherer herkunft. Häufiger ist das auslautende th $\mathrm{zu} t$ geworden (s. § 184).

Caxton kennt nur die südliche endung -eth (Römstedt 45); erst im 16. jahrhundert wird $-s$ häufig (Hoelper 54). Doch ist in Devonshire noch heute -th gebräuchlich (Ellis $157 \mathrm{ff} ., 163$ ).

\section{Pluralis präsentis.}

(§ 286) Die südliche endung -eth ist bei unseren autoren noch verhältnismässig oft zu belegen. Bei Wycliffe findet sich nicht nur beth Luk. XI 7 a, Joh. X 21 a, Hebr. V 2b, das nebst ähnlichen formen im mittellande nicht selten ist, sondern auch getith Röm. XIII $2 \mathrm{a}$, willith 1 Tim. I $7 \mathrm{~b}$; Pecock schreibt hath 150 , bildith 86 , meetith 419, fallith 488 , zeueth (?) 64 . Chaucer dagegen hat -eth weder in einsilbigen, noch in zweisilbigen wörtern; doch kennt Lydgate auch sleeth (: deth AA III 296), redithe (: dredithe 3. sing.) PS 164 . Von den übrigen Chaucerschülern hat nur Pall. beeth, ledeth, abateth, conceyveth, desireth (s. Reimindex); in der volkstümlichen litteratur finde ich belege bei Lonelich (beth : teeth XXIII 440), Beryn (doith : sothe 3669, havith : savith 3. sing. 2243), Eth. (thengep denken : lengeb länge 112), im Lamb. Ms. (doob : wroop : loob : soop II 84/26) und bei Ryman (beth : breth : deth XCII 5). In der urkundensprache finden sich nur wenige beispiele, so in London (Morsbach $134 \mathrm{ff}$.), in den späteren staatsurkunden hath $\mathrm{E} 73$, remayneth $\mathrm{E} 12$, cometh $\mathrm{R} 56$, perteyneth $R$ 83; in den Suffolker testamenten hath 1467: 16, lith 31, 
stretchith 20; in Norfolk assyngnetz N 36, longetz BL 72, hath L 66, 1451 PL I 189 . Häufiger ist -eth in Worcester, vgl. doth 408, berith 396, cheseth 395 , oweth 386 , refuseth (?) 380. Ziemlich oft erscheint -eth in den Paston Letters : hath(e) Wo I 370, 2WiP II 108, MP II 21, 83 u. ö., 1JP I 234, 2JP III (162), bethe F I 314, MP II $(124,142)$, goth u. ä. F I 174, MP I (355), doth F I (377), lyth MP II 202, owth < aggad 1JP II 211; toucheth F I (420), passyth Wo I (369), menyth Wo I 404, plesyth MP III 126, causeth I (355), standyth I (537), makyth II (124), lekyth II (125), nedythe II (140), greveth II (364), begynyth II 82, castyth 2JP II (375), wryteth II $(369,370)$, resortythe III (15), drawyth 3 JP III (55); weitere fälle bei schreibern unsicherer herkunft. Auch Caxton hat einige male -eth (Römstedt 46), und noch im 16. jahrhundert ist es nicht ausgestorben.

(§287) Sehr selten ist die nördliche endung -es; ich finde sie nur bei Lydgate (apperes : beares (pl. prs.) : yeres AA III 1678, smellys : wellys pl. : bellys pl. $\mathrm{ScPh} 887$, wohl ebenso lyues : dryues 3. sing. AG 20), bei K. Orl. (lyes : allyes : enemyes 44), im LEP (abydes : hydes häute 179, selles : felles felle 180), vKath. (lettys : mawmentys) 296 und im Lamb. Ms. (lijs : paradijs : nyce : prijs II 58/388). Die Norfolker gilden bieten dazu dreimaliges longes in BL und Wy (Schultz 39), die Paston Letters recummawnddes EP III 27, has *I (95). Auch im 16. jahrhundert ist -es noch zu belegen, vgl. die beispiele aus Skelton und Wyatt bei Hoelper s. 57, ferner suffers, keepes in Aschams Toxophilus (her. v. Arber) s. 52 und in Deckers werken (Pynson) leaps I 90, makes I 65. Noch heute finden sich im südlicheren England formen wie we knows, you aims, they deys (Ellis 93, 52, 117 u. ö.; hier wird jedoch angleichung an die 3. pers. sing. vorliegen (vgl. § 282).

(§ 288) In allen unseren denkmälern ist das gewöhnliche die mittelländische endung -en, deren konsonant jedoch oft apokopiert wird. Wycliffe pflegt sowohl bei einsilbigen (Gasner 27) wie bei zweisilbigen wörtern das $-n$ zu bewahren; nur wenn das personalpronomen dem verbum folgt, tritt wie im Ae. meist die kürzere form ein : mowe ze Matth. XII 34, zyue we 2 Kor. VI 4, serue we Hebr. XII 28, doch vndirstonden ze Mark. VII 18, gessen ze Luk. XIII 4; b bevorzugt $-n$ auch in dieser stellung. In gewöhnlicher fügung ist apokope im 
indikativ in allen teilen der Bibel recht selten; $\mathrm{K}^{1}$ bietet an beispielen honoure Dan. III 18, chese 1 Makk. IX 30, forzeue 1 Makk. XIII 39, mowe Mark. II 19, $\mathrm{K}^{2}$ wondre Joh. V 20; biseche 1 Thess. IV 1, swere Hebr. VI 16, M loue 1. Joh. III 14, b nur wole 1 Kor. X 27, bacbite 1 Petr. II 12 . Häufiger ist die endung im konj. gefallen, obgleich $-n$ auch hier das gewöhnliche ist. Vgl. aus beiden fassungen tremble Dan. VI 26, fulfille XI 14, turne Hos. V 4; honoure Joh. V 23; ponysche Röm. I 24, lyue 2 Kor. V 15, come 2 Kor. IX 5 ; telle 1 Petr. II 9 , glorifie II 12, make II 15.

(§ 289) Noch deutlicher ist die scheidung zwischen beiden modis bei Pecock. Hier ist im indikativ -en meist erhalten, sowohl bei einsilbigen (ben $2 \mathrm{u}$. oft, seen $32 \mathrm{u}$. ö., han $32 \mathrm{u}$. oft, goon 52, doon 67) wie bei zweisilbigen (senden 52, witnessen 32 , weenen 52 , seruen 52 , rehercen 32 , treten 52 ); mit abfall des $n$ sind zu belegen be $319 \mathrm{u}$. ö., mowe $2 \mathrm{u}$. oft, wole 76, dare 120, kunne 320 , schule (folgt we!) 342 , gouerne 202 , reherce 38 (konj.?); also fast nur wörter, die meist als partikeln gebraucht werden. Dagegen scheint der konjunktiv bis auf holden 91 stets apokopiert zu sein (be $39 \mathrm{u}$. oft, kepe 4, slide, stumble 75, waite 135). Das - $n$ scheint also bei Pecock fakultatives indikativzeichen $\mathrm{zu}$ sein.

(§ 290) Bei Chaucer ist apokope des $-n$ in beiden modis häufig (t. Br. § 186, 188). Bei den einsilbigen erscheint neben goon, doon, been auch bee (t. Br. § 197) und seltenes goo (Marshall-P. 62, 159). Der sprachgebrauch der Chaucerschüler lässt sich mit dem des meisters leider nicht methodisch vergleichen. Bei Chaucer können wir mit ziemlicher sicherheit den sprachgebrauch in reim und versinnerem feststellen; bei jenen sind wir fast allein auf die reime angewiesen, und in beweisenden reimen mit $-n$ erscheinen fast nur einsilbige und kontrahierte wörter (doon, goon, been, seen, lyen, sayn), deren flexionsendungen nicht immer dieselben schicksale gehabt haben wie die der zweisilbigen. Ich verzeichne daher nur die verschiedenheiten in der behandlung dieser wörter bei den Chaucerschülern. Hoccleve hat anscheinend stets die endung - $n$ (vgl. Vollmer 219); bei Lydgate ist kein wesentlicher unterschied von Chaucer nachzuweisen (doch einmal adiuren beschwören : turnen inf. AA II 1187), Bokenam hat auch do : to VI 309. Wenn in vielen dichtungen die apokopierten präsentia 
stark überwiegen, so beruht das auf ihrer grösseren reimfähigkeit (be, see reimt mit sämtlichen frz. substantiven auf -é, z. t. auch auf $-i$, vielen fürwörtern, go mit wo, fro, lo, two, no, to, so!). Die umfangreicheren volkstümlichen dichtungen verwenden beide formen des indikativs; bei Audelay finden sich auch reime mit zweisilbigem indikativ auf -en : leedon : anon : relegyon 17, deprevon: confession : passyon : electioun 24 .

(§ 291) Capgrave kennt in Kath. formen mit und ohne apokope, vgl. bene : qween II 447, han : man : kan II 1342, seene : queene : shene IV 543, seyn : ageyn I 669 ; bee : shee IV 526, doo : too II 517, lye : forby : worthy II 474, see : hee III 785, say : fray : may II 688, crye : mercye I 930, pay : day : aray V 744. In Chr. ist das $n$ bei zweisilbigen nur in drei indikativen erhalten (longyn 8, 36, writyn 132, trespasin 231), sonst endet der plur. prs. ind. und konj. stets auf $-e$, oder ist endungslos (s. § 157); die einsilbigen haben stets die endung verloren, vgl. be 1, do 9, go 43, se 124 usw.

(§ 292) An den gebrauch der Oxforder erinnert es, dass in den Londoner urkunden der indikativ häufiger die endung bewahrt hat als der konjunktiv. Im übrigen ist $-n$ in beiden modis weit seltener als $-e$; neben $b e$ erscheint jedoch ziemlich häufig ben, neben do auch doon (Morsbach $134 \mathrm{ff}$., $149 \mathrm{ff}$.). Gegen ende des jahrhunderts ist -e noch zahlreicher geworden; -en erscheint nur in den indikativen owen E 64 u. ö., followen $R 43$, causen $R 74$ gegenüber etwa 20 indikativen und 13 konjunktiven auf $-e$; be $\mathrm{R} 11,13,15 \mathrm{u}$. o. ist weit häufiger als been u. ä. $\mathrm{R} \mathrm{79,} \mathrm{83;} \mathrm{für} \mathrm{ae.} \mathrm{dō}$ erscheint nur do $\mathrm{R} 13$. Auch in Suffolk ist - $e$ das übliche; 9 mal ist $-n$ apokopiert, daneben owyn 1467: 32, folwyn 18, arn 30 u. ö., lyn 23, 30; dazu be 1467: 23 u. ö., und ebenso oft ben 1467: 17 u. ö., do 1467: 16. Der konjunktiv geht $14 \mathrm{mal}$ auf $-e$ aus. In den Norfolker gilden dagegen herrscht für den ind. -en mit ganz geringen ausnahmen, ebenso in der grossen urkunde aus der Heinrichs V (Schultz $37 \mathrm{ff}$.); in den PL dagegen erscheinen nur die indikative arn 1451 I 189, willen 1465 II 224 gegenüber sechs -e-formen; für die einsilbigen finde ich nur das beispiel ben 1451 I 190. In Worcester ist wieder -en das übliche $(10 \mathrm{mal})$; $-e$ ist nur belegt in shulle 377 , haue $379 \mathrm{u}$. o. wolle, mowe 392 (partikeln), enacte 404; dazu ben 392 u. ö., 
done 393 u. ö., gon 395 (kein do, go). Der konjunktiv hat stets das $-n$ verloren.

(§293) In den Paston Letters ist bei allen schreibern - $e$ die gewöhnliche endung beider modi. Selten findet sich -en (nur im indikativ) bei $\mathrm{F}$ : avysen I (155 u. ö.), 1WiP acorden, specifien, auch seyn, arn I 20, 25, MP waytyn I 110, k[n]owyn III 23, lewyn III 24, auch eryn II 179, 1JP owen II 222. Bei den einsilbigen mit vokalischem stammesauslaut ist die volle endung etwas häufiger: ben(e) MP I 112 u. ö., 2JP III 103 u. ö., don F I 94, MP I 112 u. ö., han 1JP II 222, doch öfter be F I 171 u. ö., MP I 178, 1JP I 348, 2JP III 103 u. ö., 3JP III 50 u. ö., $d o(o)$ MP I 112 u. ö., 2JP III 164, 3JP III 50 u. ö., se(e) F I 398, 2JP III 137, 3JP III 156, go 3JP III 50.

(§ 294) Bei Caxton (RF) ist -en noch 13 mal im indikativ erhalten (-en : -e etwa $=1: 8)$, z. b. syngen 5 , knowen 6 , stonden 9. Von einsilbigen kommen vor ben $19 \mathrm{u}$. oft, seen 114, goon 118; be 19 u. oft, goo 29 u. ö., do 19 u. oft. - Auch im heutigen Englisch ist die endung noch oft erhalten, vgl. Ellis' angaben über das nördliche mittelland s. 295, 463.

\section{Imperativ pluralis.}

(§295) Nur wenige unsrer quellen bieten ausreichende belege für diesen modus. Bei Wycliffe hat in der älteren fassung etwa ein drittel aller formen die endung -th, z. b. goth Matth. X 6, seeth Matth. XXVIII 6, heerith Sach. XI 7, greetith Kol. IV 15, holdith Offenb. II 25; in b ist stets -e dafür eingetreten, das auch in a die regel bildet. - Chaucer hat häufiger -eth als -e (t. Br. § 189), Capgrave nur die $\S 158$ aufgeführten -(e)-formen. Die früheren Londoner urkunden bieten gleich selten - $(e)$ und -eth (Morsbach $135 \mathrm{f}$.), die späteren schreiben nur faille $\mathrm{E} 67, \mathrm{R} 16$, geve $\mathrm{E} 39$, paie $\mathrm{E} 69$, demeane, entrete $\mathrm{E} 76$. Suffolk hat nur take 1467: 26, lete 37, knowe 1481: 64, wohl auch refuse, take 1467: 25; in Norfolk zeigen die spärlichen belege je einmal -eth und das nördliche $-s$, dreimal endungslosigkeit (Schultz 39f.). In den Paston Letters ist - $(e)$ die gewöhnliche endung, doch ist auch -eth nicht selten. Vgl. doth F I 338, beyth Wo I 371, wetith, porveythe AP I (59), sendyth F I (155), leythe 3JP II (348), setteth ${ }^{*}$ I (289), remembreth ${ }^{*}$ I 546, maketh ${ }^{*}$ II 214 , spekith, 
maketh Norf. I (340) usw. Einmal erscheint sendis F I 94. Bei Caxton finde ich in $\mathrm{RF}$ nur $-e$, in $\mathrm{BC}$ auch -eth (vgl. Römstedt 46).

\section{Infinitiv.}

(§ 296) Die endung -en ist in der Bibel gewöhnlich apokopiert. In den teilen nach $M$ finde ich für mehrsilbige verba überhaupt keine vollen infinitive, in $\mathrm{K}^{1}$ deuouren Dan. VII 23, heeren 1 Makk. II 22, susteynen 1 Makk. V 40 usw., preyen Matth. VI 9, welden X 9, taken X 17, resseyuen XI 14 und einige mehr, in $\mathrm{K}^{2}$ azenstonden Luk. XI 53, synnen Röm. VI 15, faylen 1 Kor. VIII 8, lyuen Gal. III 12, in A kyndlen XXXIX 9, byryen XXXIX 11 u. ö. Die jüngere fassung hat bis auf witen Ezech. XXIV 27 stets das - $n$ beseitigt. Etwas häufiger (doch, namentlich in $b$, immerhin recht selten) ist $-n$ bei den einsilbigen, vokalisch auslautenden infinitiven, z. b. ben Ezech. XL $46 \mathrm{a}$, don Luk. III $12 \mathrm{a}$, vndon Offenb. V $2 \mathrm{~b}$, goon Ezech. III 25 a.

Bei Pecock sind in auffälligem gegensatze zum plur. präs. infinitive mit voller endung überhaupt nicht mehr sicher zu belegen; auch ae. bēon (2 u. ö.), sēon (21 u. ö.), slēan (113), $d \bar{o} n$ (16 u. ö.), gōn (30), ne. flee 21 u. ö. sind stets endungslos.

(§ 297) Chaucer (t. Br. § 190) und Gower (Fahrenberg 405) haben noch ziemlich häufig die alten formen bewahrt; bei den einsilbigen erscheinen do und don, be und ben usw. Hoccleve, Lydgate und Bokenam weichen nicht ab (beachtenswert sind

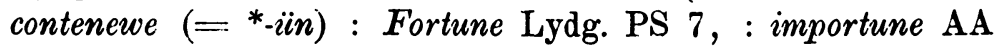
III 373, sowkyn : knelyn : Mawdelyn Bok. VIII 1095). Auffällig ist es, trotz des in $\S 290$ gesagten, dass einige werke der Chaucerschule nur oder fast nur endungslose infinitive haben, so Rich. Ros (Gröhler 17), Gen. (goo : so 832, do : to : also 933, be : she 1222, see : citee 1238 usw.), Ass. L (be : me 80, go : mo 141, see : she 299, do : so : go 415), MMgd. (go : wo : so $532 \mathrm{~b}$, do : so $533 \mathrm{a}$, be : certente $534 \mathrm{~b}$, see : blee < bleo $535 \mathrm{a})$. [Zu erwähnen ist noch heven < hebban : even < efn : seven < seofon Pall. 181/75.] Ebenso verhalten sich von den volkstümlichen dichtungen z. b. Cleges, vKath., Wolf., Bl. Hayles, Ryman. [Bemerkenswert ist striuen : azen Lamb. II 29/221.]

(§ 298) Capgrave hat in Kath. reime beiderlei art: seen : queen IV 45, done : sone IV 1123, goon : stoon V 284, auch 
seyn : pleyn Pr. 235; be : tree I 51, se : auctorite II 433, doo : too I 91, goo : woo IV 1841, say : may II 939. In Chr. erscheint -en sehr selten in mehrsilbigen wörtern: noten 48, arestin 264, anullen 281, tretyn : betyn 290 in einem gelegentlich zitierten vers, nur einmal in einem einsilbigen: sene 131, sonst heisst es stets be 2, se 10, do 30 , goo 23, sey 30 usw.

(§ 299) In den Londoner urkunden findet sich nur ein zweisilbiger infinitiv mit -en in einem alten testament, häufiger, aber auch nur in den privaturkunden, hat sich die endung in ursprünglich einsilbigen oder kontrahierten verben erhalten (Morsbach 135 ff., 149 ff.), in den späteren staatsurkunden finde ich überhaupt keinen beleg für -en oder - $n$; stets be, see $\mathrm{R} 13$, doo R 24, goo R 70 usw. In Suffolk erscheint nur eine -enform (ocupyen 1467: 31), dazu tritt der kontrahierte infinitiv lyn 1467: 23, 30. Die einsilbigen verba sind stets endungslos, so be 1481: 59 , se 1467: 30 , do 1482: 52 , go 1467: 18 . In den Norfolker gilden herrschen grosse verschiedenheiten. Einige schriftstücke (Wy) haben nie, andere (besonders die Norwicher) ganz überwiegend -en. Die einsilbigen wörter erscheinen in doppelformen. In der grossen urkunde von Norwich ist -e häufiger (-en : $-e=1: 22 / 3$ ), vgl. Schultz $36 \mathrm{ff}$., 42 . Die dokumente der Paston Letters bieten nur wenige belege für -en (sowndyn 1451 I 189, yeven 191, laboren 191) gegenüber fast $50 n$-losen infinitiven; die einsilbigen sind stets endungslos, z. b. be 1451 I 189, see 1465 II 224, doo 1459 I 462 . In Worcester ist nur einmal comyn $394 \mathrm{zu}$ belegen, sonst haben alle, auch die einsilbigen wörter, ihr $-n$ verloren.

(§300) In den Paston Letters bedient sich nur MP in grösserem umfange der vollen formen bei mehrsilbigen infinitiven: heryn, weten, lesen, entren I 109 usw.; sonst erscheinen nur noch folgende belege: abedyn 2WiP I 515 (oder = have abidden ?), gyvyn 2JP III 116, comyn < frz.comoner Wo I 370 u.ö., ferner sayn MP I 109 usw., moun (zu ae. magan) EP III 278. Die einsilbigen werden ebenso behandelt, MP schreibt ben I 109 u. oft, don 81 u. ö., gon 109 u. ö., sen II 187, (jedoch häufiger $b e$, do, go II $187 \mathrm{u}$. oft, se II 84 u. ö.), sonst erscheinen nur noch wenige $n$-formen: ben, seen F I 418, done Wo I $370,371$.

Bei Caxton bewahren mehrsilbige wörter -en weit seltener als im plur. prs., in RF kommen nur 10 fälle vor (z. b. comen 5, abhorren 9, syngen 11), bei einsilbigen sind in RF 
die vollen formen weit seltener als die apokopierten (ben 31, seen 107, goon 12, 48, doon 67, goo 48 u. oft, doo 29 u. oft, see $107 \mathrm{u}$. oft, be $29 \mathrm{u}$. oft.

\section{Partizipium präsentis.}

(§ 301) In fast allen unseren quellen ist das mittelländische suffix -ing(e) die regelmässige partizipialendung. Für Wycliffe führt Koch (Hist. Gramm. I s. 342 f.) auch das südliche -ende an, doch findet es sich nicht in den handschriften, auf die sich unsre untersuchung erstreckt. Dagegen ist bei dem Kenter Gower die endung -ende häufiger als -ing(e), vgl. Fahrenberg, 406, selten erscheint sie bei Lydgate (Schick LXXII) und Bokenam (lyuende : pretende IX 377, dredende : offende XII 252), einmal bei Lonelich (mende : comende $\mathrm{XXX} 556$, wie statt mynde : comenge zu lesen ist), ferner synkopiert im Beryn (gonde < gānde : honde 574, : withstonde 944). Häufig ist -ende in den Norfolker gilden (Schultz $36 \mathrm{ff}$.); einmal erscheint es in Exeter (acordynd 336). Die Paston Letters bieten + III 379 in einem briefe aus Yarmouth (Norfolk) besechynd; Caxton hat einmal connynd (Römstedt 46).

(§ 301 a) Auch die nördliche endung -and ist nicht gerade häufig. In der Oxforder gelehrtensprache und der Chaucerschule finde ich sie überhaupt nicht, dagegen in zwei denkmälern der volkstümlichen litteratur, Beryn (lyvand : vndirstond 1115, comand : honde 2426 , ebs. 3418 , wo comand für comyng $\mathbf{z u}$ lesen ist) und im Lambeth Ms. (hildande zu ae. hieldan beugen : hand II 23/55, pleyande : bonde < bōnda : vndirstonde : hond II 53/383). Häufiger erscheint es in Capgraves werken, vgl. lyuande : fond pt. II 9, : hande III 233 u. ö., : lande III 1178 usw., weeldande : vndirstande V 991, comande : lyuande : vndirstande V 1096 und in Chr. folowand $142 \mathrm{u}$. oft, lyand 23, lyvand 286, 315, seiand 32. An. tīpindi lautet gewöhnlich tidyndis 163 u. ö., analogische bildungen sind tydannes 242, tydyngis 203, 247, 264. Das Suffolker testament von 1482 bietet zweimal adioynand 53, 54; die Norfolker urkunden häufig -and (Schultz $36 \mathrm{ff}$.). In den Paston Letters sind zu belegen mylkand Lincoln I (98) und levand, wele wylland * II (138). 


\section{2. pers. sing. präteriti.}

(Belege fast nur bei den 0xfordern und bei Chaucer.)

(§ 302) Im starkem präteritum ist nur noch selten die ae. form auf -e mit dem ablautvokal der dritten stufe erhalten. Ziemlich häufig ersclheint were Matth. XXVI 69, Joh. I 48, Röm. XI 17, Offenbs. V 9, Ezech. XVI 6 u. ö., ferner size Offenb. XVII $8 \mathrm{a}$, corme Joh. VI $25 \mathrm{~b}$, sodann ohne -e sawz Dan. II 45 a, XIII 54 a und mit unregelmässigem ablaut spake Dan. III 36a, zaf Luk. XV 29 b, was Ezech. XVI 7 a u. ö. Gewöhnlich (namentliich in b), wird die schwache endung -st an die form der 1. 3. ssing. gehängt, so in camest Ezech. XVI 7, tokist Ezech. XVI 17 u. ö., sawist (b: siest) Dan. XIII 54 u. ö., stodist Obadja 11, swcorist Micha VII 20, wast Ezech. XVI 14 a, zauist Ezech. XVI 33 , biganst (b: bigunnest) Dan. II 29, spakist Ezech. XXXW 12 b, gatist Ezech. XXVIII 4b. Pecock bildet in der alten weise nur noch were (zitat?) 200, 262, sonst stets schwach lbarist 200, 262, camest 205, zauest 520, tookist 62, threwist 260. Bei Chaucer (t. Br. § 193) wird das pt. noch fast stets steark gebildet; auch Lydgate hat AG 1740 thou sy (: idolatry : lby), EFr I 209 thou stood : blood, MP 263/621 thou leye : dleye : preye, Bokenam thou founde : confounde usw. VIII 372, vgl. ferner thou knew : eschew RemL $538 \mathrm{~b}$, thou smote : hie wote Gen. 2351 mit ausgleich; ebs. in der volkstümlichen litteratur Lonelich thou sye : sekerly XXXIII 199, : eye XV 297, vMarg. slewe : I-nowe 352, Brompton ches : pes :: ces : reles 36, Lambeth Ms. slouz : pou I 163/51, bigan : mam : wan 2. sg. pt. : ran 2. sg. pt. II 13/39, zaue : saue inf. : crane inf. 19/41 usw. Aus Capgraves Kath. belege ich thou say : day V 1439, aus einer Norfolker urkunde von 1465 dagegen thiou heldest PL II 224. Bei Caxton wird das präteritum fast sttets schwach gebildet (Römstedt 47), doch haben noch Skelton und Tottel z.t. die alten formen bewahrt (Schöneberg 43, Hoellper 58).

(§ 303) Das schwrache präteritum hat meist die südliche endung -est, so stets: in der Bibel (preidist Matth. XVIII 32, bileuedist Joh. XX 29 usw., einmal shuldis Ezech. III 6 a) und bei Pecock (leernedisst 62, studiedist 62 usw.). Bei Chaucer ist -est ebenfalls alleiin gebräuchlich; eine endungslose 2. pers. prät. in nördlicher arrt ist bei ihm ein beabsichtigter provinzialismus (t. Br. § 1944). Doch bietet sein nachahmer K.Orl. 
thou had sad 5 ; auch in der volkstümlichen litteratur finde ich einige beispiele für dies nordenglische präteritum: soffyrd: Lord $(!=$ lauerd) Audelay 61, thou sayde (wie statt ze sayde $\mathrm{zu}$ lesen ist) : apayd 62, pou hadde : pei ledde : clad pz. : glad Lamb. I 200/554, bouzte : wrouzt pz. : pouzt : nouzt II 26/146. - Capgrave schreibt woldist 287, aber ded 75,141. In einer Norfolker urkunde von 1465 erscheint thou woldest PL II 224. Auch Caxton hat einmal thou had (Römstedt 47).

Plur. präteriti.

(§ 304) In beiden fassungen der Bibel ist bei den starken verben die endung -en gewöhnlich erhalten; beispiele für apokope sind in $\mathrm{K}^{1}$ drunke Obadja 16, toke Micha II 8, came 1 Makk. VI 46, in $\mathrm{K}^{2}$ syze Mark. IX 7, founde Mark. XIV 16, stoode Luk. XVII 13, in M were Offenb. IX 8. In b ist eine form wie ranne Mark. VI 55 ganz seltene ausnahme. Bei Pecock sind ebenfalls die apokopierten formen (nur abode 19, biganne 41, grewe 347, knewe 14, toke 30 , were $30 \mathrm{u}$. ö.) seltener als die entsprechenden unverkürzten (verhältnis $1: 2$ ), nur were ist häufiger als weren, was wohl mit dem vortonigen gebrauch des wortes zusammenhängt. Bei Chaucer ist das - $n$ oft abgefallen (t. Br. § 193). In Capgraves Chronik (in Kath. keine nennenswerten belege), verhalten sich die ursprünglichen präterita (z. b. begotin 15, foutyn 47 , ridyn 171, writyn 281) zu den apokopierten etwa wie $1: 2$. In den urkunden sind belege ziemlich spärlich. Die ältesten Londoner privatdokumente zeigen doppelt so oft -en wie - $e$; die parlamentsurkunden fast stets - $e$ (Morsbach 142); gegen ende des jahrhunderts sind nur (wenige) formen auf - $e$ zu belegen (vgl. § 160); Suffolk bietet nur holpe 1467: 28, Norfolk begunne, bore (grosse urk.), aber sowen < sāwon (Schultz 40), später were 1459 I 461 u. ö., wern 1451 I 189. In den Paston Letters ist $-n$ nur erhalten in tokyn AP I 217, abedyn MP I 111, Norf. I 199, comyn MP II 215, Norf. I 199, wern 1JP I 518 u. ö. Häufiger sind die vollen formen bei Caxton; sie verhalten sich zu denen auf -e wie 1 : 3 (beispiele smeton, camen, gauen, beten; ranne, threwe, drewe, were RF 27).

(§ 305) Bei den schwachen verben herrschen im allgemeinen analoge verhältnisse. Aus dem sprachgebrauch der Oxforder ist erwähnenswert, dass Pecock stets -en bewahrt, 
wenn der mittelvokal erhalten ist, so lyueden, seruiden, leerneden, contynueden 18. Capgrave gebraucht auffallender weise niemals mehr die vollen formen; auch in den Londoner urkunden sind sie weit seltener als beim starken verbum; schon in den ältesten schriftstücken überwiegt $-e$ (Morsbach $147 \mathrm{f}$.). Etwas häufiger als bei der starken flexion ist en in den Paston Letters, aber auch hier nur bei MP (maden I 81, haddyn u. ä., zedyn, seydyn 110, 111, seydyn III 431 neben vielen beispielen für $-e$ ), ganz vereinzelt ist seydyn Norf. I 195. Bei Caxton erscheint -en wieder verhältnismässig oft. RF bietet cryden 27 u. ö., desireden 33 , gapeden 98 , sayden 31 u. ö., gegenüber etwa 30 präteritis auf $-e$ ohne mittelvokal.

\section{Partizipium präteriti.}

(§ 306) In der Bibel endigt das pz. pt. der zweisilbigen, konsonantisch auslautenden stämme auf -en und -e; die einzelnen handschriften weichen hier stark von einander ab. In $\mathrm{K}^{1}$ ist -en bei weitem das gewöhnliche; -e findet sich in rise Ezech. XXXV 13, sowe Hos. X 13, blowe Mal. I 13 und wenigen anderen fällen. In $\mathrm{K}^{2}$ sind beide endungen etwa gleich häufig (im Lukas und Johannes -e etwas zahlreicher), z. b. takun Luk. IX 16, take Luk. X 43, knowen Joh. XIV 7, knowe Joh. XVI 3, getun 2 Kor. IV 1, gete 1 Tim. IV 6. In M dagegen ist $-e$ das gewöhnliche; weit seltener ist -en, z. b. zouun $1 \mathrm{Joh}$. V 16 u. ö., bigeten 1 Joh. IV 9, fallen Offenb. XVII 10. In A, wo allerdings die belege nicht sehr häufig sind, überwiegt -en; -e erscheint in wonne Ezech. XXVIII 4, bitake XXXI 11, forzete XXII 12. In der jüngeren fassung ist die hälfte aller verba mit beiden endungen belegt. Eine (nördliche) tendenz, - $n$ nach nasal stets zu apokopieren, lässt sich in keiner handschrift nachweisen. Die einsilbigen, vokalisch auslautenden verben werden in der älteren fassung verschieden behandelt. Stets erhalten ist das - $n$ in seen (adj. ursprungs) Baruch $\Pi 1$ I 22, Ezech. XVI 50, Gal. II 6 u. oft, stets abgefallen in be Obdj. 5, Luk. X 36, Apg. XXVII 21 u. ö., gleich häufig sind gon Matth. II 13, Luk. VII 24 und go 1 Makk. XII 27, Apg. XIII 13, don Luk. II 48, 1 Tim. V 11 u. ö. und do Joh. V 29, 2 Kor. $\mathrm{XI} 7$ u. ö. In der jüngeren fassung erscheint gleichfalls nur be Apg. XXVII 21 u. ö., doch goon Matth. II 13 neben go Luk. 
VII 24, 1 Makk. XII 27; do Luk. II 48 u. ö. ist entschieden häufiger als don Joh. XV 7 u. ö.

(§307) Bei Pecock sind ebenfalls die meisten zweisilbigen partizipia mit und ohne $-n \mathrm{zu}$ belegen. Doch heisst es fast stets bounde 18, 43,110, founde 7, 19, $48 \mathrm{u}$. oft, vndirstonde 8, 26, 27 u. oft, kunne 25, 101, 129, wonne 149, come 76, 77, $91 \mathrm{u}$. oft, nur selten bounden 18,110,494, founden 17 ; kunnen 15,16 , sungun 200. Wie bei Wycliffe erscheint nur seen 14, 99 u. oft, be 14, 29 u. oft, doon 8, 14 u. oft, do 1, 65 u. ö.

(§308) Bei Chaucer ist nach t. Br. $\S 196$ das - $n$ oft abgeworfen. Aus den reimindices Cromie und Marshall-Porter ergiebt sich noch, dass die volle endung im versauslaut oft erhalten ist, jedoch fast nur bei ursprünglich kurzsilbigen stämmen, vgl. taken, shapen, writen, byden, getyn, smetyn, dreuyn, yeuen, broken, wreken, eten (auch oft -e): dagegen heisst es stets falle, bete (bis auf je ein betyn, foldyn im Troilus), holde, songe, spronge, bounde, founde, blowe, knowe - auch stets bigonne, come, so dass in nördlicher weise nie -en nach nasal steht. Von einsilbigen erscheinen $d o$, go, bee und die entsprechenden vollen formen, stets seen. Bei Hoccleve und Lydgate ist in der behandlung der einsilbigen wörter ein unterschied nicht $\mathrm{zu}$ bemerken; Bokenam hat auch see pz. : me I 938, ebs. IX 836, XIII 1140. In den übrigen werken der Chaucerschule werden die $n$-formen auffallend bevorzugt. Nur der Gen. hat häufig be (: me 6311), doo (: twoo 6828 usw.), goo (: moo 6781), in den übrigen dichtungen findet sich nur gelegentlich einmal be (: se inf. : parde FlL 44, ebs. 309, : charite Nun 265), see (: mortalitee Wade 2245), doo (: moo : also Wade 1409), ago (: so Ass. L. 531 a). Dieselbe tendenz zeigt sich bei den volkstümlichen dichtern; see kommt überhaupt nicht vor, wenn nicht sey : cry Beryn 3079 darauf deutet.

(§ 309) Capgrave hat doppelformen wie Chaucer, in Kath. been (: qween II 1200 usw.), gon (: ston III 107 usw.), doone (: oone II 1169 usw.), iseen (: queen IV 479 usw.); be (: thre IV 1671 usw.), goo (: soo I 954 usw.), do (: so I 161 usw.). In Chr. überwiegen die apokopierten partizipia (-en : $-e=$ $1: 2^{1 / 2}$ ); an beispielen vgl. begunne 150 u. ö., bownde 150 u. ö., chose 225, come $2 \mathrm{u}$. oft, songe $65 \mathrm{u}$. ö., aber auch dronchin 225 u. ö., hangen 190 u. ö., songen 97 . Von einsilbigen erscheinen don 208, 227, 265, do 1 u. oft, go 92 u. ö., be 1, 44. 
(§ 310) In den ältesten Londoner privaturkunden überwiegt -e; -en dringt langsam vor, um etwa seit 1420 , also auch in den meisten staats- und parlamentsurkunden, regel zu werden. Von den einsilbigen schwankt $b e(n)$; don ist überall häufiger als do (Morsbach $142 \mathrm{f}$., $149 \mathrm{f}$.). Gegen ende des 15. jahrhunderts überwiegt -en noch mehr; gegenüber 17 partizipien auf - $n$ (darunter auch commen $\mathrm{R} 12$, bounden 50 , understanden 76 , founden 80 ) erscheinen mit -e nur bounde 11, come 24, founde 11 u. ö., understande 76 u. ö.; von einsilbigen findet sich nur be 13 u. ö. neben bene 13 u. ö. (usw.) mit apokope. Die Suffolker testamente behandeln das partizipium sehr ungleich. Das erste (1467) hat doppelt so viel $-e$ als -en (darunter je einmal comyn 24 ; songge 17 , bownde 25 , öfters fowunde 17 u.ö.), das zweite und vierte $(1470,1482)$ nur sehr wenige belege, das dritte (1481) 9 -en (kein beispiel mit nasal im stamm) und 4 -e (darunter bounde 61, understande 63). Unter den spärlichen belegen für einsilbige pz. erscheinen doo und doon 17, 59 u. ö., stets be 18 u. ö. Auch in Norfolk sind verschiedenheiten zu beobachten. In N, BL, WL, NL endet das pz. stets auf en (auch songen, begunnen, founden, comen), in L kommen einige ausnahmen vor (begonne, found neben en-formen, helde); 0 und $\mathrm{Wy}$, welch letzteres stark nördliches gepräge trägt, zeigen nur begunne (einsilbige pz. sind nicht belegt; Schultz 40 f.). Dagegen hat die spätere, sog. grosse urkunde von Norwich etwa gleich häufig -en und -e (darunter begonne, founde; keine derartigen formen auf - $n$; vgl. Schultz 40 ), und die schriftstücke der PL zeigen zwar 8 -en, darunter fowndyn I 189, 468; aber auch fownde I 189, take III 287 u. ö., sunge III 282, yove II 224 ; bei den einsilbigen doppelformen ohne unterschied: be II 224, do III 288 - ben, don I 189. - Die verfassung von Worcester hat $13 \mathrm{mal}$-en, darunter founden $377 \mathrm{u}$. ö., rongen 401, $3 \mathrm{mal}-e$, darunter fownde $377 \mathrm{u}$. ö., stets done 381 u. oft, ben 379 u. ö., be 381 u. ö.

(§ 311) In den Paston Letters (nur die originalbriefe sind berücksichtigt) ist die endung meist erhalten; doch ist die häufigkeit der apokope bei den einzelnen schreibern sehr verschieden. Bei $\mathrm{F}$ sind die -e-formen ziemlich häufig (founde I 418 u. ö., come I 398 u. ö., ronne I 418; aber auch bonden I 93, comyn I 92 u. ö.; begeten I 93, knowen I 398; von einsilbigen ist endungslos nur be I 172, 322); bei Wo sind die 
belege recht spärlich; $1 \mathrm{WiP}, 2 \mathrm{WiP}$ und EP haben bis auf (be)come I 25, II 108, be I 86, II 108) stets die volle endung bewahrt (kein beispiel mit nasal im stamm); MP bedient sich dagegen der verkürzten formen fast ebenso häufig wie der ursprïnglichen; auffällig ist stetes come II $203 \mathrm{u}$. ö., bownde II 185, founde II 202 u. ö., dazu be, ben II 202 u. oft, gone III 24, do ebd. u. ö. 1JP ist der einzige schreiber, der apokope bevorzugt, so holde I 518, dreve, wete I 349, doch getyn I 518; be, do I 332 u. ö., bene I 234. Fast stets ist -en erhalten bei 2JP (auch bonden III 86, comcn III $165 \mathrm{u}$. ö.); ausnahmen sind nur bownde III 86 u. ö., wone III 129; auch die einsilbigen haben bis auf goo (= ne. ago) II 221 stets $-n$. Ebenso schreibt 3JP -(e)n (kein beispiel für nasal) bis auf bownde III 290, bonde III 68, be III $41 \mathrm{u}$. ö.

(§ 312) Bei Caxton überwiegen die -cn-formen (verhältnis $3: 1$ ). Unter den beispielen sind anzufülıren: comen $6 \mathrm{u}$. oft, come 18 u. ö., wonnen, wonne 8 u. ö., bounden 45 u. ö., bounde 78 u. ö., founden 34 u. ö., founde 14 u. ö. Von den einsilbigen erscheinen endungslos nur do und be (Römstedt $50 \mathrm{f}$.).

(§ 313) Eine zusammenfassende betrachtung der endung -en zeigt zunächst, dass das $-n$ im allgemeinen am häufigsten im infinitiv, am seltensten im partizipium apokopiert wurde; auch im präteritum ist es recht häufig abgefallen, während es im plur. prs. verhältnismässig oft erhalten ist. Im grossen und ganzen ist die behandlung des $-n$ der des $-e$ analog (vgl. $§ 155 \mathrm{ff}$.). Auch zeigt sich ein bestreben, zwischen ind. und konj. plur. zu scheiden; in letzterem modus ist der abfall des $-n$ überall weiter fortgeschritten, als in ersterem; besonders deutlich erscheint diese sonderung bei Pecock.

In manchen punkten scheint die westliche gruppe konservativer zu sein, als die östliche, vgl. die behandlung des plur. prs. und prät. Caxton hat wie bei der apokope des $-e$ die ursprünglichen formen in grösserem umfange bewahrt als die etwa gleichzeitigen Londoner und Suffolker urkunden (vgl. infinitiv und präteritum).

(§ 314) Besonders auffällig sind die unterschiede in der behandlung des partizipiums präteriti. In der Oxforder gelehrtensprache schwankt -en und -e überall, wobei einzelne handschriften eine der beiden endungen sichtbar bevorzugen; in London herrscht gegen ende des 14. jahrhunderts -e, um

Anglia. N. F. XII. 
nach hundert jahren fast ganz von -en verdrängt zu werden; in Suffolk zeigt sich in der zeit von 1467-81 dieselbe erscheinung - bei unserem geringen material mag dies jedoch zufall sein; in den Norfolker urkunden ist umgekehrt -en im 14. jahrhundert fast allein gebräuchlich, im 15. jahrhundert zeigt sich eine starke minderheit von -e-formen; in Worcester ist um 1467 fast nur -en zu belegen; Caxton steht in diesem punkte den etwa gleichzeitigen urkunden der hauptstadt nahe. Ein versuch, dies auffällige durcheinander zu erklären, kommt über sehr unbestimmte vermutungen nicht hinaus. Erwähnt sei nur, dass sich nur ganz vereinzelt (Margaret Paston, Suffolk 1467?) eine tendenz geltend macht, das pz. in nördlicher weise zu gestalten; nur scheinbar gehört die sonderung von -en und $-e$ bei Chaucer hierher.

Präterito-präsentia und anomala.

(Ueber die ablantformen der prät. prs. ist $§ 250-265$ gehandelt worden.)

(§ 315) Ae. witan. An die 3. pers. sing. wird in den Paston Letters bisweilen die endung -eth der übrigen verba gehängt (vgl. die entwicklung von ae. $\bar{a} h$ ) : wo(t)tyth 3 JP II (232), III 57, 257, MgP III 295, wateth 2JP III (15), wetyth 2JP III (94), * III (182). Der infinitiv erscheint einmal als wot MP III 124 mit angleichung an den indikativ (der an dieser stelle unmittelbar vorher geht); das präteritum hat gelegentlich den stammvokal des präsens (sing., meist auch plur.) angenommen (Capgrave nur wist 139 u. ö.): wost(e) MP I 109, 112, 179 u. ö., wast 3JP III 155. Einmal lautet das pz. (w-einfluss) wust Norf. I 196. Nach dem prät. gebildet ist der imp. wist Schultz 42. - weit, wett statt wot s. § 47, 251.

(§316) Ae. cunnan. Pecock bildet ein pz. kunne(n) 15, 25, 129 u. ö., ebs. Caxton conne RF 39.

[ae. unnan. Caxton hat einmal yonned (gönnte) 66, jedoch wohl ndd. ursprungs.]

(§ 317) Ae. magan lautet im inf. stets mowe Ezech. VII 19, Offenb. VI 17, XIII 4, moun (: deuocyoun) Bok. I 773 u. ö., EP III 278. - 2. sing. mizte Luk. XVI 2 b. - Als pt. erscheint neben mihte auch mohte, vgl. mought : fought : about Lydgate AG 264, : prowt (stolz) KOrl. 101, : browhte Lon. XV 260, 468, XXI 238, : powzth OwM 298, : bote Unz. T. 24, mowht PL * III (181). 
(§ 318) Ae. mōtan. Das präteritum muste u. ä. wird bereits allgemein als präsens verwendet, so bei Pecock 87, 89, 101 u. ö., bei Capgrave 305, 308, in den urkunden (vgl. Morsbach 150, für Suffolk 1467: 26, für Norfolk PL 1451 I 189) und in den Paston Letters, so 3JP III 48 usw.; die alte präsensform mote u. ä. erscheint selten bei Capgrave $(283 ; 313$ als präteritum!) in den Norfolker urkunden (1465 PL II 224) und in den Paston Letters (MP I 112, III 29, 45 u. ö.).

(§ 319) Ae. bēon. Die übliche form für den plur. präs. ist ben (beth); daneben finde ich arn, ar(e) selten bei Chaucer (t. Br. § 197) und einigen Chaucerschülern wie K. Orl. (: square 14, : wave < wāere 103) und bei Capgrave $(17,90,306)$, in den Paston Letters (F I 398 u. ö., 1WiP I 25 u. ö., EP III 251, MP I 112 u. ö., 2JP III 165 u. ö., 3JP III 40 u. ö.), nie in den Londoner urkunden älterer und neuerer zeit, selten in Suffolk (1467: 30, 1481: 59), dagegen ziemlich oft in den Norfolker schriftstücken (Schultz 42; 1459 I 461, 1482 III 283 u. ö.) und bei Caxton (Römstedt 50). Die ae. form er (ere, err, ern, eryn) erscheint einmal in den Norf. gilden (Schultz 42) und bei MP II $179,194,195,{ }^{*}$ I (392). Dazu stellt sich ert in der älteren fassung der Bibel (Gasner 123).

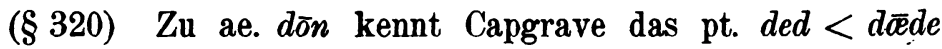
((oder dyde?) Chr. 7, 8, 20 u. oft, ebs. Caxton (Römstedt 50).

(§ 321) Ueber éode vgl. §56.

(§322) Ae. willan. Altes wellað usw. zeigt sich noch in ye nell : forswell : dwelle K. Orl. 163, wele, nel Morsbach 149, ncelyn Schultz 41, wele MP II 65. Ueber will oder woll vgl. $\$ 74$. Das pt. ist öfters nach dem prs. neugebildet: willed $\mathrm{R}$ 19 u. ö., wyld(e) 2WiP I (297), MP I (83), Devon * I (85).

(Schluss folgt.)

Berlin (Gross-Lichiterfelde). Wilhelm Dibei.ius. 